\title{
THE DEUTERIUM FRACTION IN MASSIVE STARLESS CORES AND DYNAMICAL IMPLICATIONS
}

\author{
Shuo Kong ${ }^{1}$, Jonathan C. Tan ${ }^{1,2}$, Paola Caselli ${ }^{3}$, Francesco Fontani ${ }^{4}$, Thushara Pillai ${ }^{5,6}$, Michael J. Butler ${ }^{7}$, \\ Yoshito Shimajiri ${ }^{8}$, Fumitaka NaKamura ${ }^{9}$, AND TAKeshi SAKai ${ }^{10}$ \\ ${ }^{1}$ Department of Astronomy, University of Florida, Gainesville, FL 32611, USA \\ ${ }^{2}$ Department of Physics, University of Florida, Gainesville, FL 32611, USA \\ ${ }^{3}$ Max-Planck-Institute for Extraterrestrial Physics (MPE), Giessenbachstr. 1, D-85748 Garching, Germany \\ ${ }^{4}$ INAF-Osservatorio Astrofisico di Arcetri, L.go E. Fermi 5, I-50125 Firenze, Italy \\ ${ }^{5}$ California Institute of Technology, Cahill Center for Astronomy and Astrophysics, Pasadena, CA 91125, USA \\ ${ }^{6}$ Max Planck Institut für Radioastronomie, Auf dem Hügel 69, D-53121 Bonn, Germany \\ ${ }^{7}$ Max Planck Institute for Astronomy, Königstuhl 17, D-69117 Heidelberg, Germany \\ ${ }^{8}$ Laboratoire AIM, CEA/DSM-CNRS-Universitè Paris Diderot, IRFU/Service d' Astrophysique, CEA Saclay, F-91191 Gif-sur-Yvette, France \\ ${ }_{9}$ National Astronomical Observatory of Japan, 2-21-1 Osawa, Mitaka, 181-8588 Tokyo, Japan \\ ${ }^{10}$ Graduate School of Informatics and Engineering, The University of Electro-Communications, Chofu, Tokyo 182-8585, Japan \\ Received 2015 September 29; accepted 2016 January 27; published 2016 April 15
}

\begin{abstract}
We study deuterium fractionation in two massive starless/early-stage cores, C1-N and C1-S, in Infrared Dark Cloud G028.37+00.07, which was first identified by Tan et al. with ALMA. Line emission from multiple transitions of $\mathrm{N}_{2} \mathrm{H}^{+}$and $\mathrm{N}_{2} \mathrm{D}^{+}$were observed with the ALMA, CARMA, SMA, JCMT, NRO 45 m, and IRAM $30 \mathrm{~m}$ telescopes. By simultaneously fitting the spectra, we estimate the excitation conditions and deuterium fraction, $D_{\text {frac }}^{\mathrm{N}_{2} \mathrm{H}^{+}} \equiv\left[\mathrm{N}_{2} \mathrm{D}^{+}\right] /\left[\mathrm{N}_{2} \mathrm{H}^{+}\right]$, with values of $D_{\text {frac }}^{\mathrm{N}_{2} \mathrm{H}^{+}} \simeq 0.2-0.7$, several orders of magnitude above the cosmic $[\mathrm{D}] /[\mathrm{H}]$ ratio. Additional observations of $\mathrm{o}-\mathrm{H}_{2} \mathrm{D}^{+}$are also presented that help constrain the ortho-to-para ratio of $\mathrm{H}_{2}$, which is a key quantity affecting the degree of deuteration. We then present chemodynamical modeling of the two cores, especially exploring the implications for the collapse rate relative to free-fall, $\alpha_{\mathrm{ff}}$. In order to reach the high level of observed deuteration of $\mathrm{N}_{2} \mathrm{H}^{+}$, we find that the most likely evolutionary history of the cores involves collapse at a relatively slow rate, $\lesssim$ one-tenth of free-fall.
\end{abstract}

Key words: evolution - ISM: clouds - ISM: magnetic fields - ISM: structure - stars: formation

\section{INTRODUCTION}

Massive stars produce powerful feedback that helps to shape the structure of galaxies and even the intergalactic medium. However, the formation of massive stars still involves many open questions, in part because the initial conditions of massive star birth are relatively rare, distant, and deeply embedded in massive clump/protocluster envelopes. Infrared Dark Clouds (IRDCs) are promising places to search for these initial conditions since they contain large quantities of cold $(\sim 10 \mathrm{~K})$, high density $\left(n_{\mathrm{H}} \gtrsim 10^{5} \mathrm{~cm}^{-3}\right)$ gas (e.g., Pillai et al. 2006; Rathborne et al. 2006; Butler \& Tan 2009, 2012; see review by Tan et al. 2014, hereafter T14).

Theoretically, one of the key questions is whether the formation mechanism of massive stars is a scaled-up version of low-mass star formation (Shu et al. 1987) or not. Two main competing models of massive star formation have been put forward, one is "Turbulent Core Accretion" (McKee \& Tan 2002, 2003), which is a scaled-up version of core accretion models for low-mass star formation. The other is "Competitive Accretion" (Bonnell et al. 2001; see also Wang et al. 2010). These two models involve very different initial conditions and accretion mechanisms. Turbulent Core Accretion assumes a near-virialized massive starless core for the initial condition, while Competitive Accretion forms a massive star at the center of a globally collapsing clump that fragments into a swarm of low-mass protostars. To test between the two models, it is critical to identify and characterize massive starless cores.

Once identified, it is then important to measure the virial state of a core to understand its dynamical state. The Bonnell et al. (2001) model of Competitive Accretion involves a gas cloud that is undergoing rapid collapse from a "sub-virial" state. One obstacle to determining the virial state is estimating the strength of magnetic fields. Strong magnetic fields could provide significant support in addition to other sources (mainly turbulence, since thermal pressure is dynamically unimportant in the massive, cold structures of IRDCs). However, while there is evidence for strong $B$-fields around massive protostars (e.g., Girart et al. 2009; Zhang et al. 2014), there are very few measurements at earlier stages. Recently, Pillai et al. (2015) have presented the first measurement of $B$-field strengths in dark, presumably starless regions of IRDCs, finding evidence for dynamically strong field strengths.

As an alternative approach, in this paper, we try and assess the age of a core by astrochemical indicators, in particular, the level of deuteration of key species. We compare the chemical age of a core with its dynamical (sound crossing or free-fall) timescale. If the chemical age is much greater than the dynamical timescale, then we expect that the core must have reached approximate virial equilibrium, so that if it is undergoing collapse it is at a relatively slow rate, perhaps regulated by magnetic field support.

The particular astrochemical indicator that we examine is the deuterium fraction of $\mathrm{N}_{2} \mathrm{H}^{+}\left(D_{\text {frac }}^{\mathrm{N}_{2} \mathrm{H}^{+}} \equiv\left[\mathrm{N}_{2} \mathrm{D}^{+}\right] /\left[\mathrm{N}_{2} \mathrm{H}^{+}\right]\right)$. It rises in the cold, dense conditions of starless cores, increasing by approximately three to four orders of magnitude. Theoretically, this is due to the fact that the parent exothermic reaction $\mathrm{H}_{3}^{+}+\mathrm{HD} \rightleftharpoons \mathrm{H}_{2} \mathrm{D}^{+}+\mathrm{H}_{2}+232 \mathrm{~K}$ (all in the para state; Pagani et al. 1992) is favored at low temperatures $(\sim 10 \mathrm{~K})$. Observationally, $D_{\text {frac }}^{\mathrm{N}_{2} \mathrm{H}^{+}}$has been shown to be a good evolutionary tracer for both low-mass and high-mass cores (see, e.g., Crapsi et al. 2005; Emprechtinger et al. 2009; Fontani et al. 2011; Friesen et al. 2013). Indeed, it is probably 
Table 1

Core Properties Defined by ALMA Observations of $\mathrm{N}_{2} \mathrm{D}^{+}(3-2)$ by $\mathrm{T} 13^{\mathrm{a}}$

\begin{tabular}{|c|c|c|c|c|c|c|c|c|c|c|}
\hline Core & R.A. & Decl. & $\begin{array}{l}\theta_{c}^{\mathrm{a}} \\
\left({ }^{\prime \prime}\right)\end{array}$ & $\begin{array}{c}R_{\mathrm{c}} \\
(\mathrm{pc})\end{array}$ & $\begin{array}{c}v_{\mathrm{LSR}} \\
\left(\mathrm{km} \mathrm{s}^{-1}\right)\end{array}$ & $\begin{array}{l}\sigma_{\mathrm{N}_{2} \mathrm{D}^{+}, \mathrm{obs}} \\
\left(\mathrm{km} \mathrm{s}^{-1}\right)\end{array}$ & $\begin{array}{c}\Sigma_{\mathrm{c}, \mathrm{mm}} \\
\left(\mathrm{g} \mathrm{cm}^{-2}\right)\end{array}$ & $\begin{array}{c}N_{\mathrm{H}, \mathrm{c}, \mathrm{mm}} \\
\left(10^{23} \mathrm{~cm}^{-2}\right)\end{array}$ & $\begin{array}{c}\begin{array}{c}n_{\mathrm{H}, \mathrm{c}, \mathrm{mm}} \\
\left(10^{5} \mathrm{~cm}^{-3}\right)\end{array}\end{array}$ & $\begin{array}{c}M_{\mathrm{c}, \mathrm{mm}} \\
\left(M_{\odot}\right)\end{array}$ \\
\hline C1-N & $18^{\mathrm{h}} 42^{\mathrm{m}} 46^{\mathrm{s}} .89$ & $-04^{\circ} 04^{\prime} 06^{\prime \prime} .28$ & 3.38 & 0.0818 & 81.18 & 0.367 & $0.161_{0.0938}^{0.321}$ & $0.688_{0.401}^{1.37}$ & $2.05_{1.10}^{4.12}$ & $16.2_{6.83}^{33.6}$ \\
\hline $\mathrm{C} 1-\mathrm{S}$ & $18^{\mathrm{h}} 42^{\mathrm{m}} 46^{\mathrm{s}} .50$ & $-04^{\circ} 04^{\prime} 15^{\prime \prime} 96$ & 3.61 & 0.0875 & 79.40 & 0.365 & $0.542_{0.322}^{1.08}$ & $2.31_{1.37}^{4.61}$ & $6.43_{3.52}^{12.9}$ & $62.5_{26.8}^{129}$ \\
\hline
\end{tabular}

Note.

${ }^{a}$ From the fourth column, core properties are core angular radius, core physical radius at a distance of $5 \mathrm{kpc}$, core LSR velocity as defined by $\mathrm{N}_{2} \mathrm{D}^{+}(3-2)$ emission, observed velocity dispersion of this line after accounting for hyperfine structure, mean core mass surface density estimated from $1.3 \mathrm{~mm}$ dust continuum emission, equivalent mean core column density of $\mathrm{H}$ nuclei, mean core number density of $\mathrm{H}$ nuclei, and core mass. See T13 for further discussion of these physical properties and their uncertainties.

the best tracer of pre-stellar cores, e.g., compared to $D_{\text {frac }}^{\mathrm{HNC}}$ and $D_{\text {frac }}^{\mathrm{NH}_{3}}$ (Fontani et al. 2015). Thus, overall, we consider $\mathrm{N}_{2} \mathrm{D}^{+}$to be the best diagnostic tool for detecting massive starless cores given the astrochemical model prediction of high abundance in cold, dense regions. Other methods, such as dust continuum (e.g., Rathborne et al. 2006), dust extinction (e.g., Butler \& Tan 2012), and other molecular line observations (e.g., $\mathrm{NH}_{3}$, $\mathrm{N}_{2} \mathrm{H}^{+}$), are likely subject to contamination from the much more massive clump envelope surrounding the cores. Dust continuum emission is also, in general, more sensitive to warmer, protostellar cores, rather than starless cores.

We have developed a chemical model (Kong et al. 2015) to describe the time evolution of $D_{\text {frac }}^{\mathrm{N}_{2} \mathrm{H}^{+}}$(also see Pagani et al. 2013), including for dynamical models of collapsing cores. Measurement of the abundances $\left[\mathrm{N}_{2} \mathrm{D}^{+}\right]$and $\left[\mathrm{N}_{2} \mathrm{H}^{+}\right]$, and thus $D_{\text {frac }}^{\mathrm{N}_{2} \mathrm{H}^{+}}$, in starless cores, allows estimation of core age and thus constrains the dynamical history of its collapse, e.g., the collapse rate relative to free-fall.

Two massive starless core candidates have been identified in IRDC G028.37 + 00.07 (kinematic distance of $5 \mathrm{kpc}$, Simon et al. 2006), hereafter IRDC C from the sample of BT09, from their $\mathrm{N}_{2} \mathrm{D}^{+}(3-2)$ emission observed with ALMA in Cycle 0 by Tan et al. (2003, hereafter, T13), who name the cores C1-N and C1-S. Dynamical study indicates that they are moderately subvirial, unless a relatively strong, but not exceptional, magnetic field $(\sim \mathrm{mG})$ is present. For this paper, we collected multiple lines of $\mathrm{N}_{2} \mathrm{D}^{+}$and $\mathrm{N}_{2} \mathrm{H}^{+}$from a variety of telescopes in order to estimate the excitation temperatures, column densities, and $D_{\text {frac }}^{\mathrm{N}_{2} \mathrm{H}^{+}}$. These results will then be compared with our chemical models so as to estimate core ages and constrain dynamical models.

We introduce the observational data in Section 2 and describe the measurement of $D_{\text {frac }}^{\mathrm{N}_{2} \mathrm{H}^{+}}$in Section 3. We compare to chemodynamical models to constrain core ages and collapse rates in Section 4. Discussions and conclusions are presented in Sections 5 and 6, respectively.

\section{OBSERVATIONS AND DATA REDUCTION}

The two cores were first detected by $\mathrm{N}_{2} \mathrm{D}^{+}(3-2)$ emission in ALMA Cycle 0 observations (T13). The core properties are summarized in Table 1. In this paper, we use the ALMA data from T13 (see their paper for more details of these observations). Figure 1 shows the primary beams of various observations presented in this paper, along with the two cores. Note, C1-S is away from the center of the ALMA primary beam, so we applied a primary beam efficiency correction (roughly a factor of 2.0, depending on the distance from phase center) to the observed fluxes (note, this step was not carried out in T13, where the absolute line fluxes were not utlized in the analysis; note also that there is an error in the normalization of the $y$-axis of Figure 4 of T13, which should be multiplied by a factor of $\simeq 0.5$; however, in the case of $\mathrm{C} 1-\mathrm{S}$, these two corrections effectively cancel each other out; we also note that the calibration uncertainties of these data are estimated to be $\lesssim 20 \%$ ). Those observations, in which the primary beam does not fully cover one of the cores, will not be used in the fitting analysis of that core, but we still show the corresponding spectra for reference. In summary, for $\mathrm{C} 1-\mathrm{N}$, the valid observations are $\mathrm{N}_{2} \mathrm{D}^{+}(2-1), \mathrm{N}_{2} \mathrm{D}^{+}(3-2), \mathrm{N}_{2} \mathrm{H}^{+}(1-0), \mathrm{N}_{2} \mathrm{H}^{+}(3-$ 2 ), and $\mathrm{N}_{2} \mathrm{H}^{+}(4-3)$. For $\mathrm{C} 1-\mathrm{S}$, the valid observations are $\mathrm{N}_{2} \mathrm{D}^{+}(1-0), \mathrm{N}_{2} \mathrm{D}^{+}(3-2), \mathrm{N}_{2} \mathrm{H}^{+}(1-0)$, and $\mathrm{N}_{2} \mathrm{H}^{+}(4-3)$. Below, we describe the collection of these data in detail, while Table 2 summarizes some important observational parameters.

\subsection{CARMA}

We observed the cores in $\mathrm{N}_{2} \mathrm{H}^{+}(1-0)$ at $93 \mathrm{GHz}$ with the CARMA 15-element array, using the single pointing mode. They were first observed in the D-configuration (beam size $\sim 6^{\prime \prime}, 2012$ October, bandpass calibrator: $1635+381$, phase calibrator: 1743-038, flux calibrator: Mars) and then in the Cconfiguration (beam size $\sim 3^{\prime \prime}, 2012$ December, bandpass calibrator: $1635+381$, phase calibrator: 1743-038, flux calibrator: MWC 349). Later, in 2013 August at the CARMA summer school, they were observed in the E-configuration (beam size $\sim 8^{\prime \prime}$, bandpass calibrator: $2015+372$, phase calibrator: 1743-038, flux calibrator: MWC 349). The synthesized beam is 5 ! $5 \times 4$ ! 7 with P.A. $=4^{\circ}$. The field of view is $\sim 60^{\prime \prime}$, and the largest detectable scale is $\sim 30^{\prime \prime}$ (compare to the core sizes $\lesssim 7^{\prime \prime}$ in the ALMA observation). The spectral resolution is $\sim 0.08 \mathrm{~km} \mathrm{~s}^{-1}$. The data were reduced with the MIRIAD software package. We followed the standard calibration and imaging procedures. The final $1 \sigma \mathrm{rms}$ at the map center for $\mathrm{C} 1$ is $0.050 \mathrm{Jy}_{\text {beam }}{ }^{-1}$ (combined CDE-configuration). Overall flux calibration uncertainties are estimate to be $\sim 15 \%$.

\subsection{JCMT}

We observed ortho- $\mathrm{H}_{2} \mathrm{D}^{+} J_{k^{+}, k^{-}}=1_{1,0} \rightarrow 1_{1,1}$ and $\mathrm{N}_{2} \mathrm{H}^{+}(4-$ 3) lines toward C1-N and C1-S with the JCMT $15 \mathrm{~m}$ telescope at $372 \mathrm{GHz}$ (beam size $\sim 15^{\prime \prime}$ ). We used the HARP instrument, which is a $4 \times 4$ receiver array. Each pair of adjacent receivers is separated by $30^{\prime \prime}$ and the array has a total footprint of $2^{\prime}$. The observation was carried out in the "jiggle" mode with the Nyquist sampling (1 pointing per $15^{\prime \prime}$ ). During our observation, some receivers were unavailable, so we shifted the map center so that both cores were well covered in the jiggle pattern of four adjacent working receivers (H02, H03, H04, 


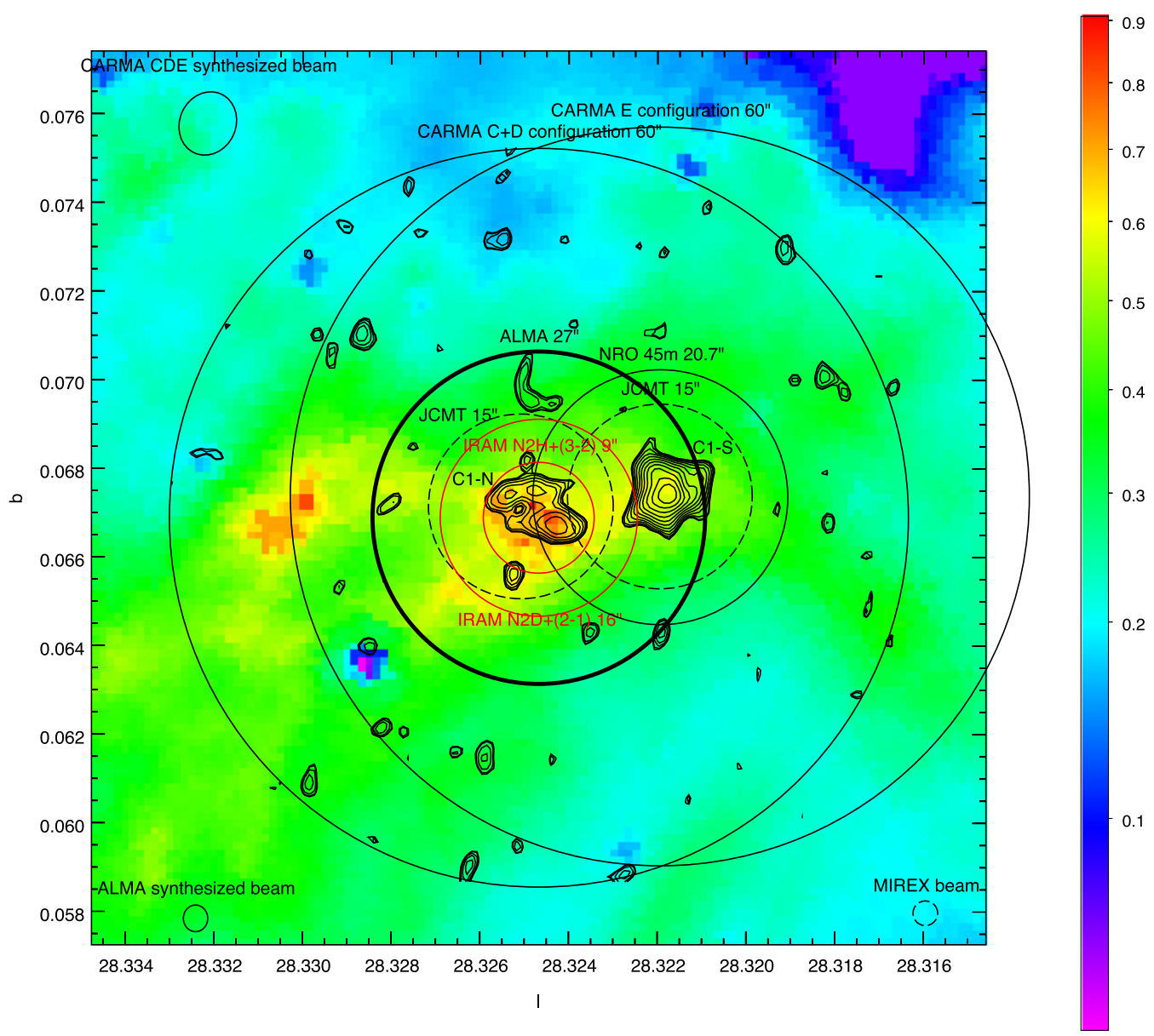

Figure 1. Observation pointings overlaid on the MIREX mass surface density map (Butler \& Tan 2012). The MIREX map is shown in log-scaled color in units of $\mathrm{g} \mathrm{cm}^{-2}$, with the 2" Spitzer beam size shown on the lower right corner. The black contours show $\mathrm{N}_{2} \mathrm{D}^{+}(3-2)$ integrated intensity from the ALMA Cycle 0 observation (Tan et al. 2013), with the synthesized beam shown in the lower left corner. The circular shapes represent the primary beams of the various telescopes used in the observations of multiple transition lines of $\mathrm{N}_{2} \mathrm{D}^{+}$and $\mathrm{N}_{2} \mathrm{H}^{+}$. Relevant telescopes and transitions are labeled next to the primary beams, respectively. The synthesized beam of CARMA data is shown on the upper left corner.

Table 2

Summary of Observations

\begin{tabular}{|c|c|c|c|c|c|c|c|}
\hline Line & Telescope & $\begin{array}{c}\text { Frequency }^{\mathrm{a}} \\
\quad(\mathrm{GHz})\end{array}$ & $\begin{array}{l}\theta^{\mathrm{b}} \\
\left({ }^{\prime \prime}\right)\end{array}$ & $\begin{array}{c}\Delta v^{\mathrm{c}} \\
\left(\mathrm{km} \mathrm{s}^{-1}\right)\end{array}$ & $\begin{array}{c}\sigma_{\mathrm{c}}^{\mathrm{d}} \\
(\mathrm{mK})\end{array}$ & $\begin{array}{c}\sigma_{s}(\mathrm{C} 1-\mathrm{N})^{\mathrm{e}} \\
(\mathrm{Jy})\end{array}$ & $\begin{array}{c}\sigma_{s}(\mathrm{C} 1-\mathrm{S})^{\mathrm{e}} \\
(\mathrm{Jy})\end{array}$ \\
\hline $\mathrm{N}_{2} \mathrm{D}^{+}(1-0)$ & NRO 45 m & 77.10924 & 22 & 0.35 & 24 & $\ldots$ & $0.038(0.7)$ \\
\hline $\mathrm{N}_{2} \mathrm{D}^{+}(2-1)$ & IRAM $30 \mathrm{~m}^{\mathrm{f}}$ & 154.21701 & 16 & 0.2 & 17 & $0.059(0.4)$ & $\ldots$ \\
\hline $\mathrm{N}_{2} \mathrm{D}^{+}(3-2)$ & ALMA $^{g}$ & 231.32183 & 2 & 0.08 & 63 & $0.046(0.08)$ & $0.082(0.08)$ \\
\hline $\mathrm{N}_{2} \mathrm{H}^{+}(1-0)$ & CARMA & 93.17340 & 5 & 0.08 & 290 & $0.050(0.32)$ & $0.056(0.32)$ \\
\hline $\mathrm{N}_{2} \mathrm{H}^{+}(3-2)$ & IRAM $30 \mathrm{~m}^{\mathrm{f}}$ & 279.51176 & 9 & 0.04 & 65 & $0.17(0.16)$ & $\ldots$ \\
\hline $\mathrm{N}_{2} \mathrm{H}^{+}(3-2)$ & SMA $^{\mathrm{h}}$ & 279.51176 & 4.5 & 0.4 & 78 & $0.17(0.8)$ & $0.20(1.6)$ \\
\hline $\mathrm{N}_{2} \mathrm{H}^{+}(4-3)$ & JCMT & 372.67249 & 15 & 0.2 & 78 & $0.75(0.8)$ & $0.49(0.8)$ \\
\hline $\mathrm{o}-\mathrm{H}_{2} \mathrm{D}^{+}(1(1,0)-1(1,1))$ & JCMT & 372.42138 & 15 & 0.2 & 78 & $\ldots$ & $\ldots$ \\
\hline
\end{tabular}

Notes.

${ }^{a}$ http://www.splatalogue.net

${ }^{\mathrm{b}}$ Angular resolution.

${ }^{c}$ Velocity resolution.

d Observation rms in $T_{\mathrm{mb}}$.

${ }^{\mathrm{e}}$ Spectra rms in flux density unit after binning (velocity resolution after binning shown in parentheses with units of $\mathrm{km} \mathrm{s}^{-1}$ ), “...” indicates that the data is not used in spectral fitting.

${ }^{\mathrm{f}}$ From Fontani et al. (2011).

${ }^{\mathrm{g}}$ From T13.

${ }^{\mathrm{h}}$ From T. Pillai et al. (2016, in preparation). 
$\mathrm{H} 05,1^{\prime}$ spatial coverage). We made sure the most massive core C1-S was at one of the pointing centers. The system temperature was $286 \mathrm{~K}$. We adopt a main-beam efficiency of 0.64 . The observations were carried out during the best weather condition at JCMT, with $\tau_{225}<0.05$ and had a pointing error of less than $3^{\prime \prime}$ on average. We obtained the calibrated data and used the Starlink software package to co-add and re-grid the data to construct the cube. The final sensitivity is shown in Table 2. The overall flux calibration uncertainties are estimate to be $\lesssim 20 \%$.

\subsection{Nobeyama $45 \mathrm{~m}$}

The Nobeyama $45 \mathrm{~m}$ observations were conducted in May 2013 toward the $\mathrm{C} 1-\mathrm{S}$ core. We observed $\mathrm{N}_{2} \mathrm{D}^{+}(1-0)$ at $77 \mathrm{GHz}$, with a beam size of $\sim 22^{\prime \prime}$. The data were taken in the position-switching mode. The $\mathrm{TZ}$ receiver was used in combination with the Fast Fourier Transform Spectrometer (SAM45), providing a bandwidth of $63 \mathrm{MHz}$ and a frequency resolution of $15.26 \mathrm{kHz}$ (corresponding to $0.05 \mathrm{~km} \mathrm{~s}^{-1}$ at the observing frequency). The pointing was checked by observing the IRC $+00363 \mathrm{SiO}$ maser emission every $1 \mathrm{hr}$, and was shown to be accurate within a few arcseconds. The main-beam efficiency was $53.4 \%$. During the observation, the system noise temperature was around $170-220 \mathrm{~K}$. The final sensitivity is shown in Table 2. Overall flux calibration uncertainties are estimate to be $\sim 10 \%$.

\subsection{IRAM $30 \mathrm{~m}$}

The IRAM 30 m data of $\mathrm{N}_{2} \mathrm{D}^{+}(2-1)$ and $\mathrm{N}_{2} \mathrm{H}^{+}(3-2)$ presented in this paper are taken from Fontani et al. (2011). Their spectra in main-beam temperature have been converted to flux density (following Section 3.3 Equation (1)). The overall flux calibration uncertainties are estimate to be $\sim 20 \%$. These observations were pointed at $\mathrm{C} 1-\mathrm{N}$, so we only include them in the analysis of this core. However, in the $\mathrm{N}_{2} \mathrm{D}^{+}(2-1)$ spectrum, there are two velocity components, and one of them corresponds to the system velocity of $\mathrm{C} 1-\mathrm{S}$. This is consistent with the fact that the IRAM $30 \mathrm{~m} \mathrm{~N}_{2} \mathrm{D}^{+}(2-1)$ observation has a primary beam partially covering $\mathrm{C} 1-\mathrm{S}$ (see Figure 1 ). Given the $\sim 1.8 \mathrm{~km} \mathrm{~s}^{-1}$ system velocity difference between $\mathrm{C} 1-\mathrm{N}$ and $\mathrm{C} 1$ $\mathrm{S}$ and the good velocity resolution of the data, we are able to isolate the two cores in velocity space. To remove the flux contribution from the $\mathrm{C} 1-\mathrm{S}$ component (blue) wing of the $\mathrm{C} 1-\mathrm{N}$ spectrum, we fit the two velocity components with the CLASS software, ${ }^{11}$ and subtract the $\mathrm{C} 1-\mathrm{S}$ velocity component. Again, the IRAM $30 \mathrm{~m}$ data are not used in the C1-S analysis.

\subsection{SMA}

SMA observations were made as part of the "SMA survey of high-mass starless cores" in the most compact configuration (sub-compact) in two tracks at $279 \mathrm{GHz}$ in 2007 to 2008. The observations were done in track-sharing mode with multiple sources per track. The correlator was configured for a uniform spectral resolution of $\sim 0.4 \mathrm{~km} \mathrm{~s}^{-1}$ at $279 \mathrm{GHz}$. Typical system temperatures were between 150 and $250 \mathrm{~K}$. The gain calibrators were J1733-130, J1911-201, and J1743-038. The bandpass calibrator was either $3 \mathrm{C} 273$ or $3 \mathrm{C} 454.3$, whichever source was brighter. The flux calibrators were Uranus, Callisto, and Titan. The overall flux calibration uncertainties are

\footnotetext{
${ }^{11}$ http://www.iram.fr/IRAMFR/GILDAS
}

estimate to be $\sim 15 \%$. The synthesized beam is $5^{\prime \prime} \times 4^{\prime \prime}$. Further details on the observing and imaging will be reported in a separate publication on the survey (Pillai et al. 2016, in preparation).

\section{RESULTS}

\subsection{Structure of $\mathrm{N}_{2} \mathrm{H}^{+}$Emission around the $\mathrm{N}_{2} \mathrm{D}^{+}$Cores}

Figure 2(a) shows the integrated intensity imaging of the $\mathrm{C} 1$ region by ALMA in $\mathrm{N}_{2} \mathrm{D}^{+}(3-2)$, CARMA in $\mathrm{N}_{2} \mathrm{H}^{+}(1-0)$, and JCMT in $\mathrm{N}_{2} \mathrm{H}^{+}$(4-3). The ALMA $\mathrm{N}_{2} \mathrm{D}^{+}$(3-2) cores are located within a filament of $\mathrm{N}_{2} \mathrm{H}^{+}(1-0)$ emission. However, the $A L M A$ cores appear to be offset from the local CARMA $\mathrm{N}_{2} \mathrm{H}^{+}(1-0)$ peaks by $\simeq 3$ !" 6 , corresponding to 18,000 au or 0.1 pc at $5 \mathrm{kpc}$. The map of $\mathrm{N}_{2} \mathrm{H}^{+}$(4-3) shows a peak that is offset to higher Galactic latitudes from C1-S by $\sim 7$ !' 2 (or $0.2 \mathrm{pc}$ ). Figure 2 (b) shows the integrated intensity map from the SMA observation of $\mathrm{N}_{2} \mathrm{H}^{+}(3-2)$. There is a peak of emission relatively close to the $\mathrm{C} 1-\mathrm{S}$ core, but again offset by about one core radius. There is a less pronounced concentration of emission toward $\mathrm{C} 1-\mathrm{N}$.

These results, especially the $\mathrm{N}_{2} \mathrm{H}^{+}$(4-3) map, suggest that there is an extended envelope of relatively warm gas around the cores. Locally high volume density is not likely to be the reason for the $\mathrm{N}_{2} \mathrm{H}^{+}$(4-3) peak, since such a volume density peak should be associated with a dust continuum peak, which is not apparent in the $1.3 \mathrm{~mm}$ emission maps of T13. The $\mathrm{N}_{2} \mathrm{H}^{+}$(4-3) peak does not seem to be associated with high column density, as seen in the morphology of the mass surface density map in Figure 1, where a clear decreasing gradient can be seen from $b=0.068$ to $b=0.072$. Rather, it seems more likely that the peak of $\mathrm{N}_{2} \mathrm{H}^{+}$(4-3) emission is caused by a local volume of gas with higher temperature. Since it is at the edge of the cloud, it might be caused by moderate shocks from external gas flows or the dissipation of turbulence in the area. Wang et al. (2006) reported a water maser detection in this area (outside C1-S's lowest contour), though at a different velocity $\left(59.5 \mathrm{~km} \mathrm{~s}^{-1}\right)$ and in single channel $\left(0.66 \mathrm{~km} \mathrm{~s}^{-1}\right)$. However, this water maser was not detected in the more sensitive observations of Chambers et al. (2009). If it was a real detection, it may be linked to shock-heated gas in the envelope. We note that Pon et al. (2015) have detected $\mathrm{CO}(8-7)$ and (9-8) emission toward the $\mathrm{C} 1-\mathrm{N}$ and $\mathrm{S}$ cores and argue that it is likely that this emission results from turbulence dissipating in low velocity shocks, rather than being due to photo-dissociation region heating.

Figures 2(c)-(f) show the first moment maps (relative to $v_{\mathrm{LSR}}$ of $\mathrm{C} 1-\mathrm{S}$ ) of $\mathrm{N}_{2} \mathrm{H}^{+}(1-0)$ (isolated hyperfine component), $\mathrm{N}_{2} \mathrm{H}^{+}(3-2)$ (full HFS), $\mathrm{N}_{2} \mathrm{H}^{+}(4-3)$ (full HFS), and $\mathrm{N}_{2} \mathrm{D}^{+}(3-2)$ (full HFS), respectively. The $\mathrm{C} 1-\mathrm{N}$ and $\mathrm{S}$ cores are surrounded by $\mathrm{N}_{2} \mathrm{H}^{+}$emitting gas that has broadly the same radial velocity as that of the $\mathrm{N}_{2} \mathrm{D}^{+}(3-2)$ from the cores, although the $\mathrm{N}_{2} \mathrm{H}^{+}(3-$ 2) mean velocity around $\mathrm{C} 1-\mathrm{S}$ is blueshifted by a few $\mathrm{km} \mathrm{s}^{-1}$, probably due to the presence of another velocity component, discussed below. We notice a relatively large velocity gradient $\left(\sim 1 \mathrm{~km} \mathrm{~s}^{-1} /\left(2 R_{\mathrm{c}}\right) \sim 6 \mathrm{~km} \mathrm{~s}^{-1} \mathrm{pc}^{-1}\right)$ in $\mathrm{N}_{2} \mathrm{H}^{+}(1-0)$ emission across $\mathrm{C} 1-\mathrm{S}$. This velocity gradient does not seem to be influenced by the nearby presence of $\mathrm{C} 1-\mathrm{N}$, which is located in a direction that is orthogonal to that of the gradient. The $\mathrm{N}_{2} \mathrm{H}^{+}$(4-3) emission also shows a gradient across the position of $\mathrm{C} 1-\mathrm{S}$ (though with much lower resolution), but the direction is different and seems likely to be caused by the $\mathrm{C} 1-\mathrm{N}$ to $\mathrm{C} 1-\mathrm{S}$ axis. 

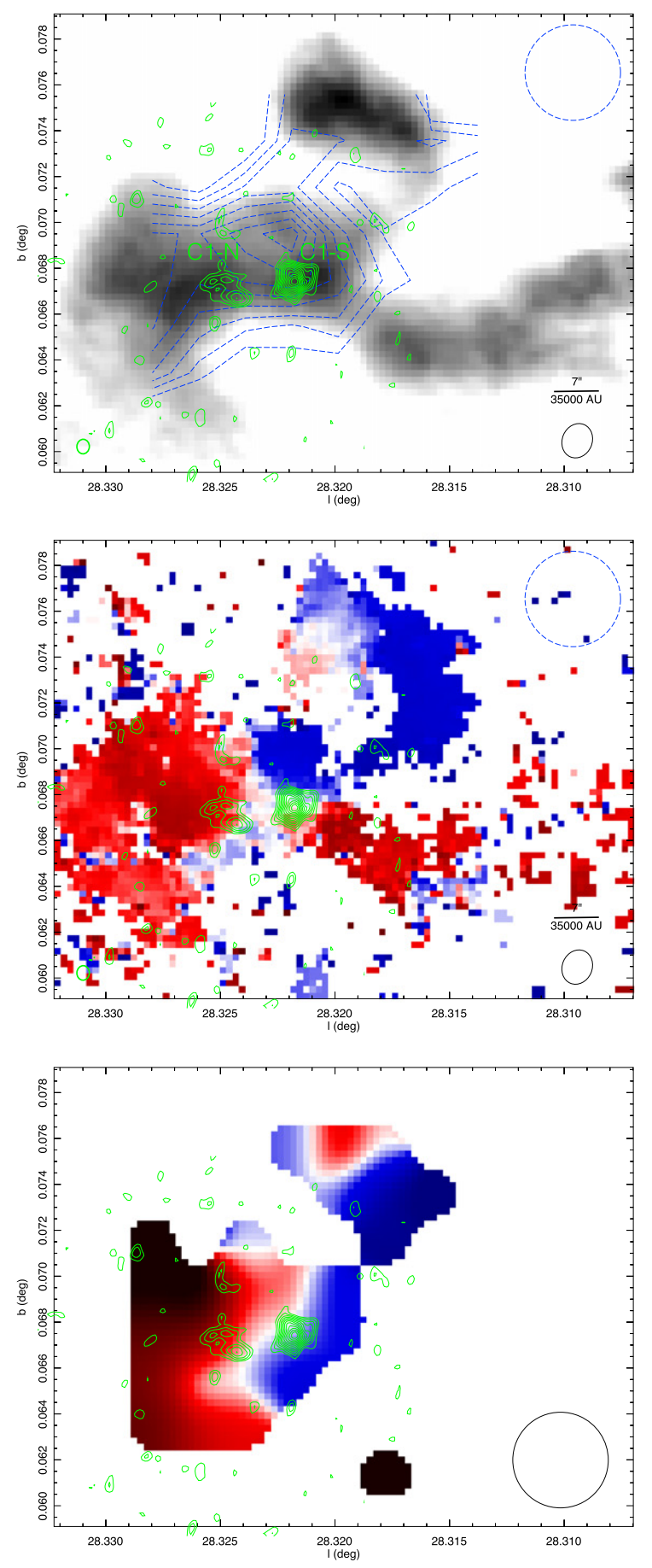
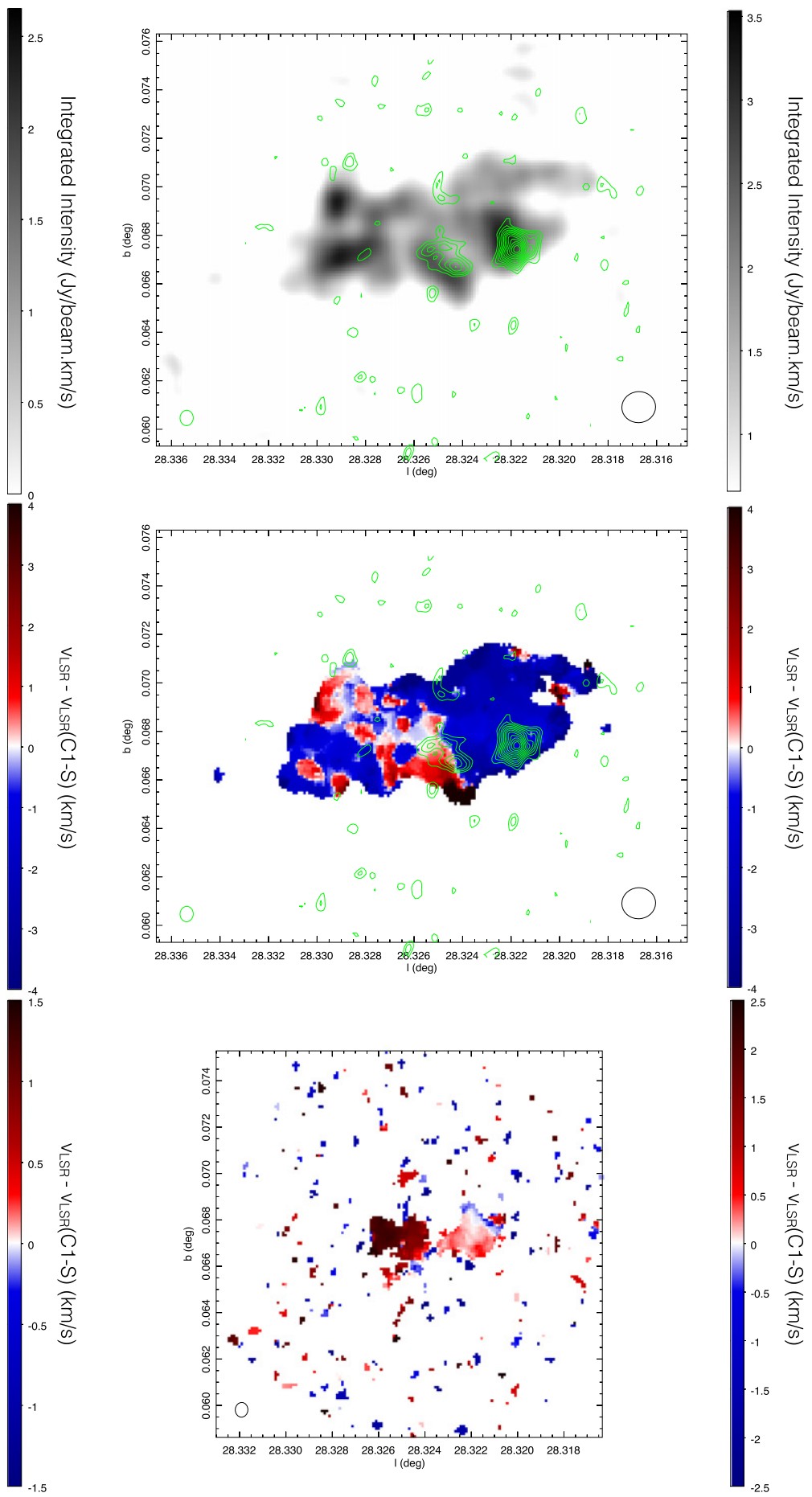

Figure 2. (a) Top left: integrated intensities of $\mathrm{N}_{2} \mathrm{H}^{+}(1-0)$ (gray-scale in units of $\mathrm{Jy} \mathrm{beam}^{-1} \mathrm{~km} \mathrm{~s}^{-1}$, with intensities integrated over velocity range $v_{\mathrm{LSR}}=68-90 \mathrm{~km} \mathrm{~s}^{-1}$, i.e., including all hyperfine structure (HFS); only pixels with $\mathrm{S} / \mathrm{N}>2$ are shown; noise at the map center is $0.09 \mathrm{Jy}^{\mathrm{beam}}{ }^{-1} \mathrm{~km} \mathrm{~s}^{-1}$ and at the map edge is $0.16 \mathrm{Jy}_{\text {beam }}^{-1} \mathrm{~km} \mathrm{~s}^{-1}$; CARMA beam is in the lower right), $\mathrm{N}_{2} \mathrm{H}^{+}$(4-3) (blue dashed contours from $3 \sigma$ to $10 \sigma$, with the noise level being $\sigma=0.10 \mathrm{~K} \mathrm{~km} \mathrm{~s}^{-1}$, with intensities integrated over velocity range $v_{\mathrm{LSR}}=75-84 \mathrm{~km} \mathrm{~s}^{-1}$ to cover full HFS; JCMT beam is shown in upper right), $\mathrm{N}_{2} \mathrm{D}^{+}(3-2)$ showing $\mathrm{C} 1-\mathrm{N}$ and S cores reported by T13 (green contours from 2, 3, $4 \ldots 14 \sigma$ with $\sigma=0.0109 \mathrm{Jy} \mathrm{beam}^{-1} \mathrm{~km} \mathrm{~s}^{-1}$, with intensities integrated over velocity range $v_{\mathrm{LSR}}=76.8-81.9 \mathrm{~km} \mathrm{~s}^{-1}$, covering full HFS; ALMA beam is in the lower left). (b) Top right: integrated intensities of $\mathrm{N}_{2} \mathrm{H}^{+}(3-2)$ (gray-scale in units of Jy beam ${ }^{-1} \mathrm{~km} \mathrm{~s}^{-1}$, with intensities integrated over velocity range $v_{\mathrm{LSR}}=68-90 \mathrm{~km} \mathrm{~s}^{-1}$, i.e., including all HFS; only cells with $>2 \sigma$ signal are shown, with $\sigma=0.33$ Jy beam ${ }^{-1} \mathrm{~km} \mathrm{~s}^{-1}$; SMA beam is in the lower right) and $\mathrm{N}_{2} \mathrm{D}^{+}(3-2)$ (green contours; same as in (a)). (c) Middle left: first moment map of the $\mathrm{N}_{2} \mathrm{H}^{+}(1-0)$ isolated hyperfine component, showing velocities in $\mathrm{km} \mathrm{s}^{-1}$ relative to $v_{\mathrm{LSR}}(\mathrm{C} 1-\mathrm{S})$. CARMA beam is in the lower right. The $\mathrm{N}_{2} \mathrm{D}^{+}(3-2)$ integrated intensity green contours are shown as in (a), highlighting the C1-N and S cores. (d) Middle right: first moment map of the $\mathrm{N}_{2} \mathrm{H}^{+}(3-2)$ total HFS, showing velocities in km s${ }^{-1}$ relative to $v_{\mathrm{LSR}}(\mathrm{C} 1-$ $\mathrm{S})$. SMA beam is in the lower right. The $\mathrm{N}_{2} \mathrm{D}^{+}(3-2)$ integrated intensity green contours are shown as in (a), highlighting the C1-N and $\mathrm{S}$ cores. (e) Bottom left: first moment map of $\mathrm{N}_{2} \mathrm{H}^{+}(4-3)$ emission, showing velocities in $\mathrm{km} \mathrm{s}^{-1}$ relative to $v_{\mathrm{LSR}}(\mathrm{C} 1-\mathrm{S})$ (integrating over full HFS structure). JCMT beam is in the lower right. The $\mathrm{N}_{2} \mathrm{D}^{+}$(3-2) integrated intensity green contours are shown as in (a), highlighting the C1-N and S cores. (f) Bottom right: first moment map of $\mathrm{N}_{2} \mathrm{D}^{+}(3-2)$ emission, showing velocities in $\mathrm{km} \mathrm{s}^{-1}$ relative to $v_{\mathrm{LSR}}(\mathrm{C} 1-\mathrm{S})$ (integrating over full HFS structure). ALMA beam is in the lower left. 
In summary, the larger-scale kinematics around $\mathrm{C} 1-\mathrm{N}$ and $\mathrm{S}$ are relatively complex and it seems likely that $\mathrm{N}_{2} \mathrm{H}^{+}(1-0)$, (3-2) and (4-3) emission may be dominated by (or at least have significant contributions from) gas components that are separate from the $\mathrm{N}_{2} \mathrm{D}^{+}(3-2)$ cores. This will affect our method for estimating the deuteration fraction in the cores, effectively meaning that we can only use the $\mathrm{N}_{2} \mathrm{H}^{+}(1-0)$, (3-2), and (4-3) spectra extracted from the core locations to place upper limits on the level of such emission from the cores. In particular, it is the $\mathrm{N}_{2} \mathrm{H}^{+}(1-0)$ data from CARMA and the $\mathrm{N}_{2} \mathrm{H}^{+}(3-2)$ data from SMA that are most constraining, since they have the most comparable angular resolutions as the ALMA observation of $\mathrm{N}_{2} \mathrm{D}^{+}(3-2)$. Figures 2(a) and (b) indicate that $\mathrm{N}_{2} \mathrm{H}^{+}(1-0)$ and (32) spectra extracted from the location of the $\mathrm{C} 1-\mathrm{N}$ and $\mathrm{S}$ cores may have $\sim 50 \%$ flux contributions from a larger-scale clump envelope.

\subsection{Spectra of $\mathrm{N}_{2} \mathrm{D}^{+}$and $\mathrm{N}_{2} \mathrm{H}^{+}$Emission toward the Cores}

With the above considerations in mind, we proceed to analyze the $\mathrm{N}_{2} \mathrm{H}^{+}$and $\mathrm{N}_{2} \mathrm{D}^{+}$spectra extracted from locations of the C1-N and C1-S cores (i.e., for the CARMA and SMA data, these are apertures based on the ALMA $\mathrm{N}_{2} \mathrm{D}^{+}(3-2)$ core sizes from $\mathrm{T} 13$ and listed in Table 1; for the single-dish observations, these are from locations centered on the cores, or otherwise as close to the core positions as allowed). These spectra are shown in Figure 3 for C1-N and Figure 4 for C1-S.

An examination of the spectra indicate that different kinematic features can be present among the different tracers. For example, the $\mathrm{N}_{2} \mathrm{H}^{+}$(4-3) spectra show different kinematics from the $\mathrm{N}_{2} \mathrm{D}^{+}(3-2)$ cores. We perform a hyperfine structure fitting to the $\mathrm{N}_{2} \mathrm{H}^{+}(4-3)$ spectrum in C1-S using the HFS method in CLASS, and the results show that the velocity width in C1-S is $1.3 \mathrm{~km} \mathrm{~s}^{-1}$, much wider than the $\mathrm{N}_{2} \mathrm{D}^{+}(3-2)$ spectra $\left(\sim 0.5 \mathrm{~km} \mathrm{~s}^{-1}\right.$, see panels (c) and (f) in Figure 4 for C1-S). The C1- $\mathrm{N} \mathrm{N}_{2} \mathrm{H}^{+}(4-3)$ spectrum is more complicated since it shows two peaks, with one being at the C1-N $v_{\mathrm{LSR}}$ and the other being at roughly the C1-S $v_{\mathrm{LSR}}$. A fit to the velocity components gives a $0.76 \mathrm{~km} \mathrm{~s}^{-1}$ width for $\mathrm{C} 1-\mathrm{N}$, moderately larger than the C1-N N ${ }_{2} \mathrm{D}^{+}(3-2)$ spectra $\left(\sim 0.5 \mathrm{~km} \mathrm{~s}^{-1}\right.$, see panels (c) and (f) in Figure 3 for $\mathrm{C} 1-\mathrm{N}$ ). These results are also suggestive that the $\mathrm{N}_{2} \mathrm{H}^{+}(4-3)$ line in the $\mathrm{C} 1-\mathrm{S}$ and $\mathrm{C} 1-\mathrm{N}$ regions mostly traces warmer gas in an envelope external to the cores. The relatively high velocity dispersion could be caused by shocks. In the following sections, we use the spectra to constrain the excitation temperatures and deuteration fractions in the cores.

\subsection{Excitation Temperatures, Column Densities, and Deuterium Fractions of the $\mathrm{N}_{2} D^{+}$Cores}

Here we utilize the $\mathrm{N}_{2} \mathrm{D}^{+}$core models of T13, i.e., two spherical cores $\mathrm{C} 1-\mathrm{N}$ and $\mathrm{C} 1-\mathrm{S}$, with the properties listed in Table 1. The excitation temperature of $\mathrm{N}_{2} \mathrm{D}^{+}$, column densities of $\mathrm{N}_{2} \mathrm{D}^{+}$and $\mathrm{N}_{2} \mathrm{H}^{+}$and thus $D_{\text {frac }}^{\mathrm{N}_{2} \mathrm{H}^{+}}$of the cores will be constrained by the multitransition observations of $\mathrm{N}_{2} \mathrm{D}^{+}$and $\mathrm{N}_{2} \mathrm{H}^{+}$in the following way. We construct models that match the spectra of $\mathrm{N}_{2} \mathrm{D}^{+}(3-2)$, i.e., assuming all of this emission comes from the cores. These models then make predictions for the other transitions. For optically thin conditions, the modeled flux from the cores cannot exceed the observed flux; however, the modeled flux may be less than that observed if there is a contribution from a surrounding envelope, either from emission along the line of sight or from larger angular scales if the cores are unresolved (i.e., as in the single-dish observations).

The $\mathrm{N}_{2} \mathrm{D}^{+}(1-0),(2-1)$, and (3-2) and $\mathrm{N}_{2} \mathrm{H}^{+}(1-0),(3-2)$, and (4-3) spectra are shown with black lines in Figure 3 for C1-N and Figure 4 for C1-S. For the ALMA, CARMA, and SMA data, the fluxes are directly extracted from the cores since they are resolved. For single-dish data, spectra in main-beam temperature $T_{\mathrm{mb}}$ are converted to $S_{\nu}$ using

$$
S_{\nu}=T_{\mathrm{mb}} \frac{2 k \Omega_{\mathrm{mb}}}{\lambda^{2}}
$$

where $\Omega_{\mathrm{mb}}$ is the main-beam solid angle, and $k$ is Boltzmann's constant. All observed spectra have peak signal-to-noise ratios (S/Ns) $>5$, if necessary, achieved by smoothing in velocity. The resulting noise level in each velocity channel is listed in Table 2.

First, we model the $\mathrm{N}_{2} \mathrm{D}^{+}$lines to obtain the best fit for $T_{\mathrm{ex}}$, which is needed for estimating total column densities. When performing the multitransition fitting of the contribution of the C1-N and C1-S cores to the $\mathrm{N}_{2} \mathrm{D}^{+}$spectra, we make two assumptions: (1) all hyperfine components are optically thin (this will be checked by radiative transfer modeling, below); (2) all hyperfine components have the same Gaussian profile velocity dispersion, $\sigma_{\mathrm{N}_{2} \mathrm{D}^{+}}$. All hyperfine components are summed to obtain the blended model spectra, which are to be compared with the observed spectra after normalization of the integrated intensity and velocity dispersion, which are set by the ALMA-observed $\mathrm{N}_{2} \mathrm{D}^{+}(3-2)$, since this defines the cores of interest. Note, the ALMA observation has the best sensitivity, it resolves the cores, and it filters out emission from large-scale structures $\left(>9^{\prime \prime}\right)$.

We vary $T_{\mathrm{ex}}$ to reproduce the flux in $\mathrm{N}_{2} \mathrm{D}^{+}(2-1)$ and $\mathrm{N}_{2} \mathrm{D}^{+}(1-$ 0 ) from the single-dish observations as closely as possible, but making sure the model spectra do not exceed the observed ones. Since these two $\mathrm{N}_{2} \mathrm{D}^{+}$lines are observed at relatively low angular resolution, emission from the envelope and/or other larger-scale structures may be contributing.

Panels (a)-(c) of Figures 3 and 4 show model spectra with a variety of $T_{\mathrm{ex}}$ (and thus a variety of total column densities of $\mathrm{N}_{2} \mathrm{D}^{+}$) with green and red lines. Note that since the ALMA $\mathrm{N}_{2} \mathrm{D}^{+}(3-2)$ line sets the normalization of equivalent width and velocity dispersion, the green lines in panel (c) overlap closely with this observed spectrum. Also note that for $\mathrm{N}_{2} \mathrm{D}^{+}(1-0)$ of C1-N (panel (a) of Figure 3) and $\mathrm{N}_{2} \mathrm{D}^{+}(2-1)$ of C1-S (panel (b) of Figure 4), we do not have good observed spectra since the core is just outside the primary beam. While we display these spectra here for reference (since they may have some contribution from the cores), we do not use them to constrain the model spectra.

The best-fit models are shown with solid green lines and the derived values of $T_{\mathrm{ex}}$ and $N_{\mathrm{N}_{2} \mathrm{D}^{+}}$are listed in Table 3. We derive best-fit excitation temperatures $\sim 4 \mathrm{~K}$ and column densities of $\sim 6 \times 10^{12} \mathrm{~cm}^{-2}$ for both $\mathrm{C} 1-\mathrm{N}$ and $\mathrm{C} 1-\mathrm{S}$. We refer to these estimates as "Case 1." To estimate the uncertainty caused by noise, we consider a range of models about the best-fit value that is allowed by the $1 \sigma \mathrm{rms}$ noise of the spectra, i.e., for its constraint on the height of the peak of the model spectrum. These errors are listed in parentheses in Table 3.

The derived values of $T_{\mathrm{ex}}$ are $\sim 2 \mathrm{~K}$ lower than those adopted by Fontani et al. (2011; $6.4 \mathrm{~K})$, based on hyperfine fitting to $\mathrm{N}_{2} \mathrm{H}^{+}(3-2)$ single-dish observations. As discussed earlier, $\mathrm{N}_{2} \mathrm{H}^{+}$ appears to trace a wider and presumably warmer envelope 

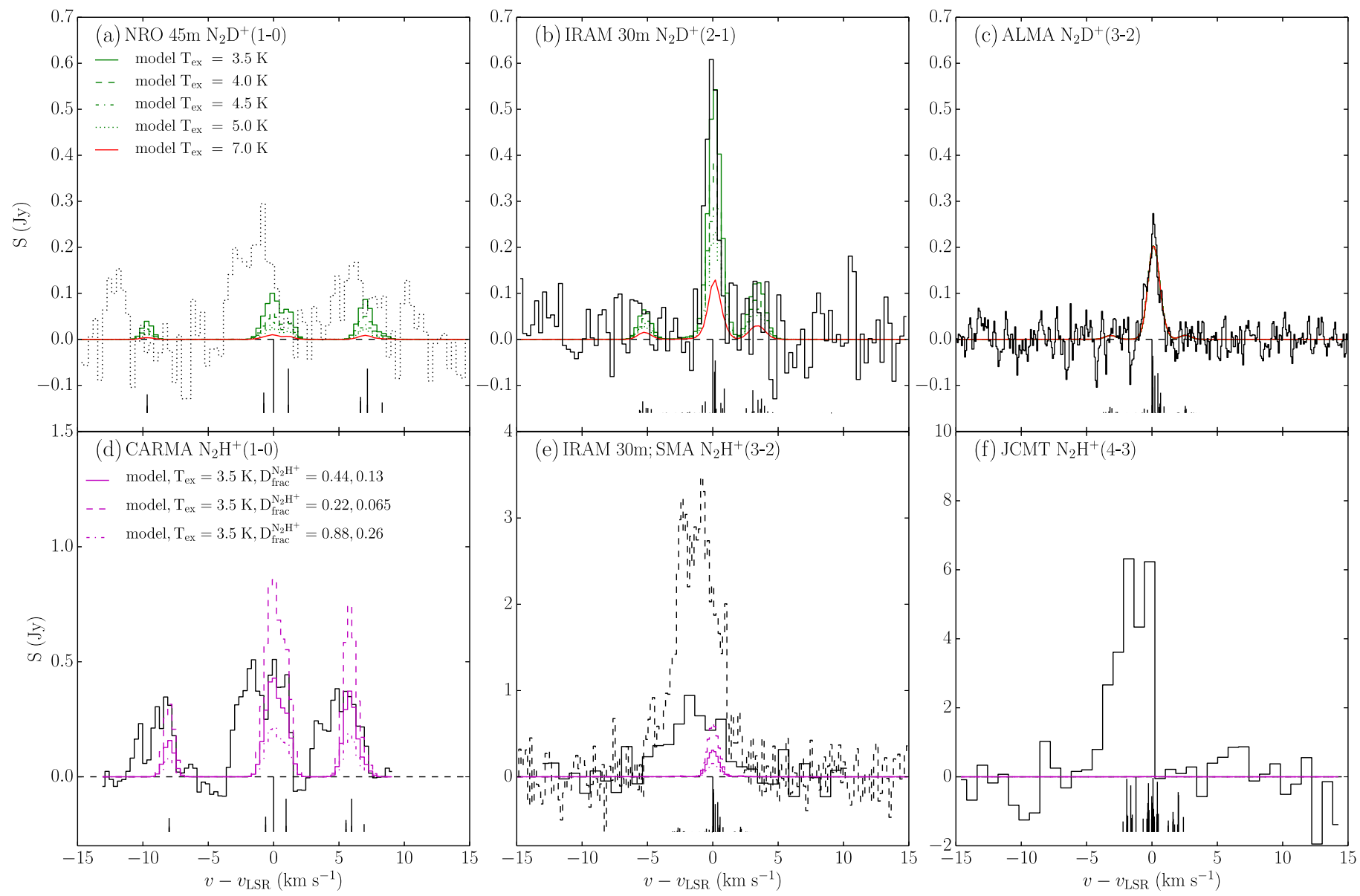

Figure 3. Upper row, panels (a)-(c): observed $\mathrm{N}_{2} \mathrm{D}^{+}(1-0)$, (2-1), (3-2) flux density spectra for C1-N (black lines), all shown in the rest frame of C1-N's $v_{\mathrm{LSR}}$ (Table 1). The normalized HFS intensities are shown underneath each spectrum, also in this velocity frame. After smoothing, the observed spectra all have peak $\mathrm{S} / \mathrm{N}>5$. The resulting spectral resolutions and $1 \sigma$ noise levels are listed in Table 2 . The model $\mathrm{N}_{2} \mathrm{D}^{+}$spectra, normalized by the ALMA $\mathrm{N}_{2} \mathrm{D}^{+}(3-2)$ emission, are shown with green and red lines with various values of $T_{\text {ex }}$ (see the legend). Note, the $\mathrm{N}_{2} \mathrm{D}^{+}(1-0)$ data (dotted black line in panel (a)) is not used for constraining the model because the NRO $45 \mathrm{~m}$ observation was centered on C1-S. In Fontani et al. (2011), the $\mathrm{N}_{2} \mathrm{D}^{+}(2-1)$ spectrum has two major velocity components, with the lower velocity component being $-1.8 \mathrm{~km} \mathrm{~s}^{-1}$ away (i.e., from C1-S). We fit hyperfine structures to the spectra and subtract the C1-S component, leaving the spectrum for C1-N shown in panel (b). Lower row, panels (d)-(f): $\mathrm{N}_{2} \mathrm{H}^{+}$(1-0), (3-2) (SMA—solid line; IRAM $30 \mathrm{~m}$-dashed line), (4-3) flux density spectra for C1-N (black lines), again all having peak $\mathrm{S} / \mathrm{N}>5$. Modeled $\mathrm{N}_{2} \mathrm{H}^{+}$spectra are shown with magenta lines for various values of $D_{\text {frac }}^{\mathrm{N}_{2} \mathrm{H}^{+}}$(see the legend, which shows Case 1 and 2 values, see the text). The normalized HFS intensities are shown underneath each spectrum (note, the $\mathrm{N}_{2} \mathrm{H}^{+}(4-3) \mathrm{HFS}$ have $60 \%$ flux in the central group).

region compared to $\mathrm{N}_{2} \mathrm{D}^{+}$. Deuterated species are likely to trace colder conditions (e.g., Caselli et al. 1999; Pagani et al. 2013; Kong et al. 2015). Note that Crapsi et al. (2005) measured $T_{\mathrm{ex}}$ to be about $4.5 \mathrm{~K}$ in a number of low-mass cores, only slightly larger than our derived values. However, it is also possible that our result of a relatively low $T_{\text {ex }}$ may be explained by the fact that we are fitting two $\mathrm{N}_{2} \mathrm{D}^{+}$lines, with the lower transition being observed by a single-dish telescope that receives some flux from regions just beyond the $\mathrm{N}_{2} \mathrm{D}^{+}(3-2)$-defined cores.

Our estimates of $T_{\mathrm{ex}}$ are relatively low compared to expected kinetic temperatures of pre-stellar cores, i.e., $\gtrsim 6 \mathrm{~K}$ (Crapsi et al. 2007, for L1544). The dust temperature in C1-N and S is constrained to be $\lesssim 13 \mathrm{~K}$, from the fact that these regions appear dark at 70 and even $100 \mu \mathrm{m}$ (T13). At the high densities of the cores, we would expect gas and dust temperatures to be reasonably well coupled. Still, subthermal excitation of the $\mathrm{N}_{2} \mathrm{D}^{+}$lines is a possibility, even though the average volume densities are close (within a factor of a few) to the critical density of the $\mathrm{N}_{2} \mathrm{D}^{+}(3-2)$ transition.

Since there are reasons to expect that our above Case 1 estimates for $T_{\mathrm{ex}}$ may be lower limits due to flux contamination from extended envelopes, as a "Case 2" estimate, we will also consider higher values of $T_{\text {ex }}$. One possible upper limit is $\sim 10 \mathrm{~K}$, set by the dust temperature. However, we note that adopting $T_{\mathrm{ex}}=10 \mathrm{~K}$ results in a negligible amount of flux in the $\mathrm{N}_{2} \mathrm{D}^{+}(1-0)$ line, which we consider to be inconsistent with the NRO $45 \mathrm{~m}$ observations of C1-S. Caselli et al. (2003) adopted a kinetic temperature of $\sim 7 \mathrm{~K}$ in L1544. We will use this value of $T_{\mathrm{ex}}$ for the Case 2 models, which are shown by the red lines in Figures 3 and 4.

To derive the $\mathrm{N}_{2} \mathrm{H}^{+}$column density in a core (and thus $D_{\text {frac }}^{\mathrm{N}_{2} \mathrm{H}^{+}}$), we assume that this species has the same value of $T_{\mathrm{ex}}$ as $\mathrm{N}_{2} \mathrm{D}^{+}$(for the $4 \mathrm{~K}$ case, if $\mathrm{N}_{2} \mathrm{H}^{+}$has a higher temperature by $1 \mathrm{~K}$, then this would increase the estimate of $\mathrm{N}_{2} \mathrm{H}^{+}$and $D_{\text {frac }}^{\mathrm{N}_{2} \mathrm{H}^{+}}$ by $30 \%$ ). However, as shown in Section $3.1, \mathrm{~N}_{2} \mathrm{H}^{+}$lines show very extended emission around $\mathrm{C} 1-\mathrm{N}$ and $\mathrm{C} 1-\mathrm{S}$. In addition, the temperature of the envelope gas could differ from those in the cores, likely being higher. Therefore, flux from the $\mathrm{N}_{2} \mathrm{H}^{+}$ envelope is likely to be contributing to (and perhaps dominating) the spectra, especially in the single-dish observations of higher $J$ transitions.

Therefore, in fitting the model of core emission to the $\mathrm{N}_{2} \mathrm{H}^{+}$ spectra, we assume the best fit is achieved when the peak flux 

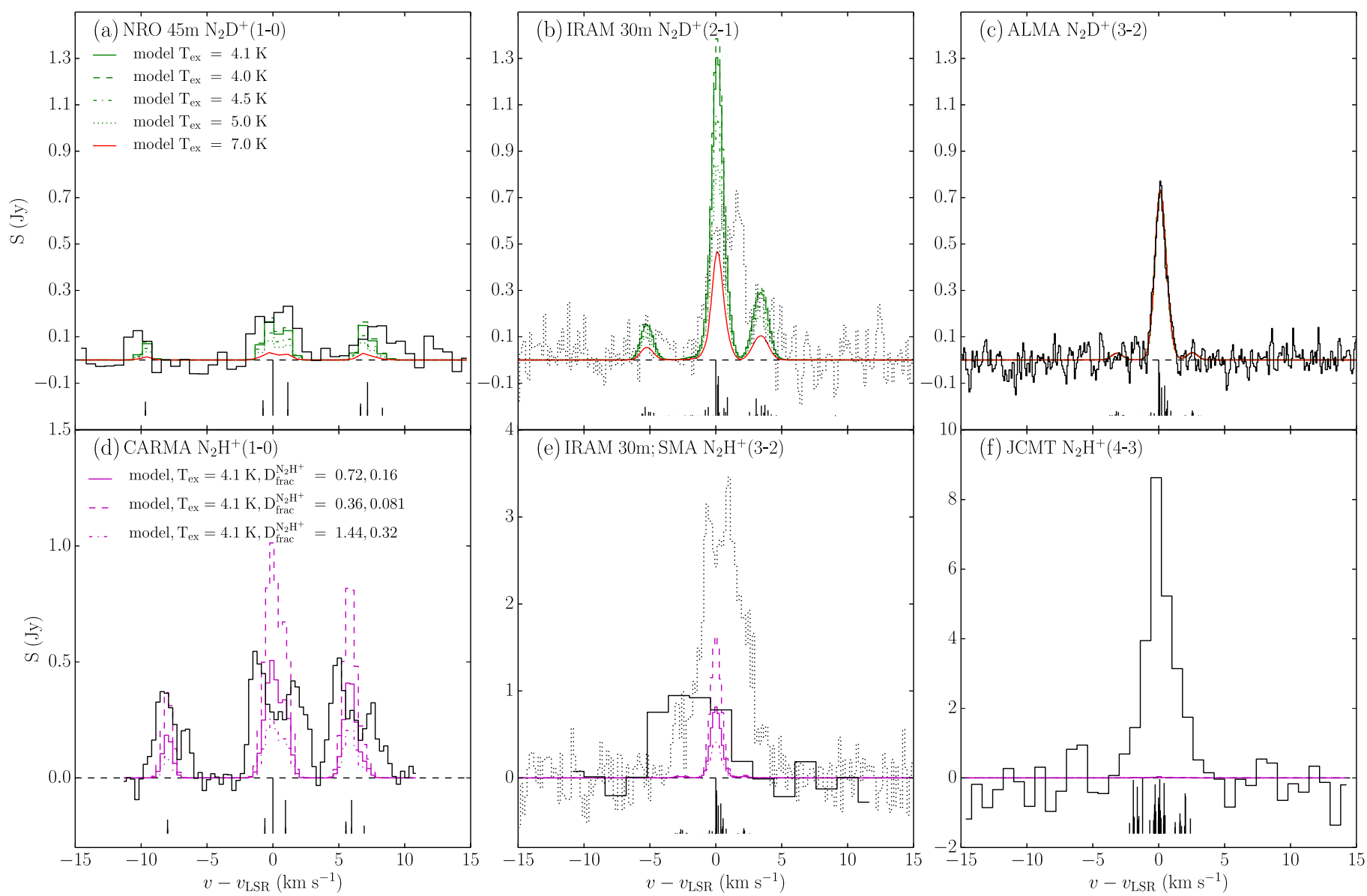

Figure 4. Same as Figure 3, but for C1-S. Again, the smoothed velocity resolution and relevant $1 \sigma$ noise level are shown in Table 2 . IRAM $30 \mathrm{~m}$ observations of $\mathrm{N}_{2} \mathrm{H}^{+}$(3-2) and $\mathrm{N}_{2} \mathrm{D}^{+}(2-1)$, shown with dotted lines, are not used to constrain the modeling because their pointings were centered on C1-N.

Table 3

Excitation Temperatures, Column Densities, and Deuterium Fraction

\begin{tabular}{lccccr}
\hline \hline Model & Core & $\begin{array}{c}T_{\mathrm{ex}}{ }^{\mathrm{a}} \\
(\mathrm{K})\end{array}$ & $\begin{array}{c}N_{\mathrm{N}_{2} \mathrm{D}^{+}} \\
\left(10^{13} \mathrm{~cm}^{-2}\right)\end{array}$ & $\begin{array}{c}N_{\mathrm{N}_{2} \mathrm{H}^{+}{ }^{\mathrm{b}}} \\
\left(10^{13} \mathrm{~cm}^{-2}\right)\end{array}$ \\
\hline Case 1 & C1-N & $3.50(0.16)$ & $0.56(0.20)$ & $0.63-\mathbf{1 . 2 6}(\mathbf{0 . 2 6})-2.52$ & $0.22-\mathbf{0 . 4 4}(\mathbf{0 . 1 0})-0.88$ \\
& C1-S & $4.12(0.22)$ & $0.59(0.21)$ & $0.41-\mathbf{0 . 8 2}(\mathbf{0 . 1 4})-1.64$ & $0.36-\mathbf{0 . 7 2}(\mathbf{0 . 1 5})-1.4$ \\
\hline Case 2 & C1-N & 7.0 & 0.029 & $0.065-\mathbf{0 . 1 3}-0.26$ & $0.081-\mathbf{0 . 1 6}-0.32$ \\
& C1-S & 7.0 & 0.083 & $0.28-\mathbf{0 . 5 5}-1.11$ & $0.075-\mathbf{0 . 1 5}-0.30$ \\
\hline
\end{tabular}

Notes. The bold numbers are used to highlight the central values.

${ }^{\mathrm{a}}$ Derived from $\mathrm{N}_{2} \mathrm{D}^{+}$fitting.

${ }^{\mathrm{b}}$ Central values based on fitting to $50 \%$ of the observed $\mathrm{N}_{2} \mathrm{H}^{+}(1-0)$ isolated component, with error in parentheses based on noise; range set by assuming $25 \%$ to $100 \%$ of this flux (see the text).

density of the model spectrum reaches the observed flux density, which in practice will be constrained by the isolated component of the CARMA $\mathrm{N}_{2} \mathrm{H}^{+}(1-0)$ spectrum and the SMA $\mathrm{N}_{2} \mathrm{H}^{+}(3-2)$ observation. For the $\mathrm{N}_{2} \mathrm{H}^{+}(1-0)$ emission, compared to the main hyperfine component group at $v-v_{\mathrm{LSR}} \sim 0 \mathrm{~km} \mathrm{~s}^{-1}$, the isolated component (at negative relative velocity) is more likely to be optically thin. Also, given the considerations of Section 3.1, we expect only $\sim 50 \%$ of the flux of the observed $\mathrm{N}_{2} \mathrm{H}^{+}(1-0)$ spectra to come from the $\mathrm{N}_{2} \mathrm{D}^{+}$core, with the rest coming from the clump envelope. However, we will consider a range of $25 \%-100 \%$, i.e., a factor of two above or below the central value, as an inherent uncertainty in this estimate, which will thus translate into a similar uncertainty in the derived
$N_{\mathrm{N}_{2} \mathrm{H}^{+}}$and $D_{\mathrm{frac}}^{\mathrm{N}_{2} \mathrm{H}^{+}}$. Given the relatively poorer sensitivity of the SMA $\mathrm{N}_{2} \mathrm{H}^{+}(3-2)$ observations, we will use this as a consistency check for the above fitting procedure. We note that these $\mathrm{N}_{2} \mathrm{H}^{+}(3-2)$ spectra show a significant velocity spread to negative velocities (also consistent with the observations of Chen et al. 2010), which indicates that additional kinematic components that are separate from the $\mathrm{N}_{2} \mathrm{D}^{+}(3-2)$ cores could be contributing flux even at the systemic velocities of the cores.

Panels (d)-(f) of Figures 3 and 4 show the observed (black lines) and core model (magenta lines) $\mathrm{N}_{2} \mathrm{H}^{+}$spectra. From the relative heights of the $\mathrm{N}_{2} \mathrm{H}^{+}(1-0)$ hyperfine groups, we see that the main group components are likely to be affected by optical depth. The best-fit models (assuming 50\% of the $\mathrm{N}_{2} \mathrm{H}^{+}(1-0)$ 

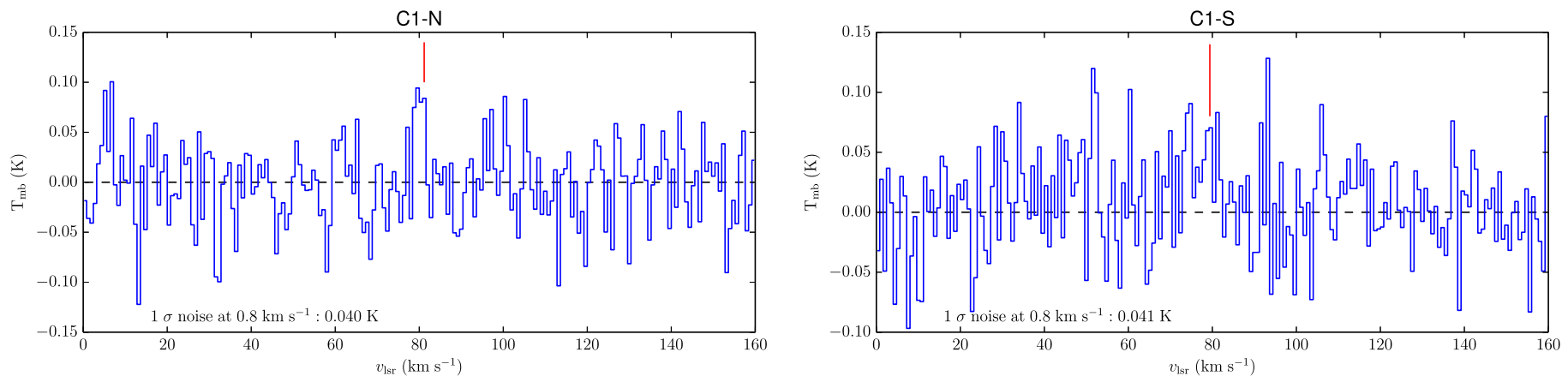

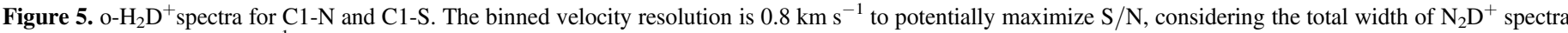
from ALMA are $\sim 0.9 \mathrm{~km} \mathrm{~s}^{-1}$. The red vertical lines mark the $v_{\mathrm{LSR}}$ of the cores. rms noise levels are labeled in the figure panels.

isolated component comes from the core) are shown with solid lines, and the relevant derived column densities of $\mathrm{N}_{2} \mathrm{H}^{+}$and thus values of $D_{\text {frac }}^{\mathrm{N}_{2} \mathrm{H}^{+}}$are listed in Table 3 .

To check the optically thin assumption, we calculate the optical depth of line emission from the model cores using RADEX $^{12}$ (van der Tak et al. 2007). The common input parameters are kinetic temperature, $T_{k}=10 \mathrm{~K}$, and line width, $\Delta v=0.9 \mathrm{~km} \mathrm{~s}^{-1}$. Then, for $\mathrm{C} 1-\mathrm{N}$, we set the $\mathrm{H}_{2}$ number density $n_{\mathrm{H}_{2}}=1.02 \times 10^{5} \mathrm{~cm}^{-3}$ and the $\mathrm{N}_{2} \mathrm{H}^{+}$column density $N_{\mathrm{N}_{2} \mathrm{H}^{+}}=2.52 \times 10^{13} \mathrm{~cm}^{-2}$, yielding maximum optical depths for the $\mathrm{N}_{2} \mathrm{H}^{+}(1-0)$ (isolated component), $\mathrm{N}_{2} \mathrm{H}^{+}(3-2)$ and $\mathrm{N}_{2} \mathrm{H}^{+}$(4-3) lines of $0.54,0.40$, and 0.016 , respectively. Similarly, for $\mathrm{C} 1-\mathrm{S}$, we set $n_{\mathrm{H}_{2}}=3.21 \times 10^{5} \mathrm{~cm}^{-3}$ and $N_{\mathrm{N}_{2} \mathrm{H}^{+}}=1.64 \times 10^{13} \mathrm{~cm}^{-2}$, yielding maximum optical depths of $0.21,0.39$, and 0.029 , respectively, for these same lines. We expect that $\mathrm{N}_{2} \mathrm{D}^{+}$lines are less affected by self-absorption than the $\mathrm{N}_{2} \mathrm{H}^{+}$lines, given that their column density is a factor of a few smaller. The estimated optical depths are relatively small, with the largest effect being for $\mathrm{N}_{2} \mathrm{H}^{+}(1-0)$. Given the uncertainties in core structure that preclude the construction of an accurate radiative transfer analysis, for simplicity, we continue with our assumed optically thin modeling results, but acknowledge that a correction for $\mathrm{N}_{2} \mathrm{H}^{+}(1-0)$ optical depth would lead to smaller estimates of $D_{\text {frac }}^{\mathrm{N}_{2} \mathrm{H}^{+}}$by a factor of about 0.6 for $\mathrm{C} 1-\mathrm{N}$ and 0.8 for $\mathrm{C} 1-\mathrm{S}$.

As discussed above, for the higher $J \mathrm{~N}_{2} \mathrm{H}^{+}$lines, the model core spectra only reproduce small fractions of the total observed flux, which is likely due to there being a dominant contribution from larger-scale, warmer envelope gas. To illustrate the excitation conditions that are needed for the higher $J$ emission, we calculate the line ratios between $\mathrm{N}_{2} \mathrm{H}^{+}(3-$ 2) and $\mathrm{N}_{2} \mathrm{H}^{+}$(4-3) seen in the spectra of Figures 3 and 4 and compare with results from RADEX models. The models explore a grid of physical conditions, with $10^{5} \mathrm{~cm}^{-3} \leqslant n_{\mathrm{H}} \leqslant 10^{6} \mathrm{~cm}^{-3}, 5 \mathrm{~K} \leqslant T_{k} \leqslant 30 \mathrm{~K}$, and other fixed parameters, including $\quad N_{\mathrm{N}_{2} \mathrm{H}^{+}}=10^{13} \mathrm{~cm}^{-2}$, velocity width $=1.0 \mathrm{~km} \mathrm{~s}^{-1}$. The best-fitting models have $T_{k} \simeq 28 \mathrm{~K}$, with the majority of models requiring $T_{k} \gtrsim 20 \mathrm{~K}$. Since the kinetic temperature $T_{k}$ is only at most $\sim 13 \mathrm{~K}$ in the $\mathrm{C} 1-\mathrm{N}$ and $\mathrm{C} 1-\mathrm{S}$ cores (T13), this supports the interpretation that this emission comes from warmer, perhaps shock-heated, envelope regions.

\footnotetext{
12 http://www.sron.rug.nl/ vdtak/radex/radex.php
}

\section{4. $\mathrm{o}-\mathrm{H}_{2} \mathrm{D}^{+}$Abundance}

Figure 5 shows the JCMT-observed $\mathrm{o}-\mathrm{H}_{2} \mathrm{D}^{+}$spectra for $\mathrm{C} 1$ $\mathrm{N}$ and $\mathrm{C} 1-\mathrm{S}$. To maximize $\mathrm{S} / \mathrm{N}$, we binned the spectra to have $0.8 \mathrm{~km} \mathrm{~s}^{-1}$ spectral resolution, considering that the line widths of $\mathrm{N}_{2} \mathrm{D}^{+}(3-2)$ are $\sim 0.9 \mathrm{~km} \mathrm{~s}^{-1}$. There is no obvious detection around $v_{\mathrm{LSR}}$ of the cores at a level of $3 \sigma$. However, over a few channels close to these $v_{\mathrm{LSR}}$ values, there is a tendency for there to be a lack of negative $T_{\mathrm{mb}}$, which may indicate a tentative detection. We follow Caselli et al. (2003) to calculate the column density. The $\mathrm{o}-\mathrm{H}_{2} \mathrm{D}^{+}$excitation temperature is uncertain. Caselli et al. (2003) assumed LTE and adopted $T_{\text {ex }}=7 \mathrm{~K}$ (i.e., the value of $T_{k}$ in L1544). In T13, we estimated $T_{k} \lesssim 10 \mathrm{~K}$ from dust temperature. However, $T_{k}$ could be as low as $6 \mathrm{~K}$ in some low-mass cores (e.g., Crapsi et al. 2007). Here we adopt a range of $T_{\text {ex }}$ from $4 \mathrm{~K}$ (allowing for subthermal excitation) to $10 \mathrm{~K}$ and set $T_{\mathrm{ex}}=7 \mathrm{~K}$ as a fiducial value. Then we divide the column density of $\mathrm{o}-\mathrm{H}_{2} \mathrm{D}^{+}$by $N_{\mathrm{H}}$ (estimated from the millimeter continuum in T13, see Table 1) to obtain the abundance of $\mathrm{o}-\mathrm{H}_{2} \mathrm{D}^{+}$. This results in a band of $\left[0-\mathrm{H}_{2} \mathrm{D}^{+}\right]$ upper limits. For $\mathrm{C} 1-\mathrm{N}$, this band is from $2.4 \times 10^{-11}$ to $7.3 \times 10^{-10}$. For $\mathrm{C} 1-\mathrm{S}$, the range is from $0.72 \times 10^{-11}$ to $2.3 \times 10^{-10}$. Note that the values of these upper limits are uncertain by at least a factor of several, given $T_{\text {ex }}$ and $N_{\mathrm{H}}$ uncertainties. Later, we will use these results to constrain astrochemical models.

Caselli et al. (2003) measured $\left[0-\mathrm{H}_{2} \mathrm{D}^{+}\right]$in $\mathrm{L} 1544$ to be $5.5-10 \times 10^{-11}$, depending on the assumption of $T_{\mathrm{ex}}$ (note that we have expressed abundances relative to $\mathrm{H}$ nuclei, rather than $\mathrm{H}_{2}$ ). These values for L1544 happen to be within our estimates of the $3 \sigma$ upper limits in $\mathrm{C} 1-\mathrm{N}$ and $\mathrm{C} 1-\mathrm{S}$.

\section{CHEMODYNAMICAL MODELING}

We run astrochemical models developed by Kong et al. (2015, hereafter K15) to compare with the above observational results. The goal is to obtain the most probable collapse rates for $\mathrm{C} 1-\mathrm{N}$ and $\mathrm{C} 1-\mathrm{S}$.

\subsection{The Fiducial Case}

The astrochemical models from K15 follow gas phase spin state chemistry of all relevant three-atom species along with $\mathrm{H}_{3} \mathrm{O}^{+}$and deuterated isotopologues (which are important for $\mathrm{O}$ chemistry). K15 also include time-dependent depletion/ desorption (TDD) of heavy elements onto dust grains, starting from some initial assumed depletion factor, $f_{D, 0}$, of heavy elements. 
K15 modeled dynamical density evolution (DDE), involving the core density at time $t$ evolving as

$$
\frac{d n_{\mathrm{H}}(t)}{d t}=\alpha_{\mathrm{ff}} \frac{n_{\mathrm{H}}(t)}{t_{\mathrm{ff}}(t)}
$$

where $t_{\mathrm{ff}}$ is the local free-fall time at current density $n_{\mathrm{H}}$, and $\alpha_{\mathrm{ff}}$ is a dimensionless parameter setting the collapse rate. We consider a "look-back" time, $t_{\text {past }}$, relative to the present time, $t_{1}$, i.e., related by

$$
t_{\text {past }}=t_{1}-t .
$$

So the density at $t_{\text {past }}$ is described by

$$
n_{\mathrm{H}, \mathrm{past}}=n_{\mathrm{H}, 1}\left[1+3.60 \alpha_{\mathrm{ff}}\left(\frac{n_{\mathrm{H}, 1}}{10^{5} \mathrm{~cm}^{-3}}\right)^{1 / 2}\left(\frac{t_{\text {past }}}{10^{6} \text { year }}\right)\right]^{-2} \text {, }
$$

where $n_{\mathrm{H}, 1}$ is the present-day density of the core, which we will define by observation.

We adopt the core density estimated from dust millimeter emission in T13, i.e., $n_{\mathrm{H}, 1}=n_{\mathrm{H}, \mathrm{c}, \mathrm{mm}}$. We have $n_{\mathrm{H}, 1}(\mathrm{C} 1-$ $\mathrm{N})=2.0 \times 10^{5} \mathrm{~cm}^{-3} \quad$ and $n_{\mathrm{H}, 1}(\mathrm{C} 1-\mathrm{S})=6.4 \times 10^{5} \mathrm{~cm}^{-3}$ (Table 1), with uncertainties of about a factor of two. The other initial conditions and fiducial parameter values are a fixed kinetic temperature of $10 \mathrm{~K}$ (see the fiducial value of $15 \mathrm{~K}$ in K15), a cosmic-ray ionization rate of $2.5 \times 10^{-17} \mathrm{~s}^{-1}$; an initial density that is 10 times smaller than the current density, i.e., $n_{\mathrm{H}, 0}=0.1 n_{\mathrm{H}, 1}$, an initial depletion factor of $\mathrm{C}, \mathrm{N}, \mathrm{O}$ from the gas phase of $f_{D, 0}=3$, and an initial $\mathrm{OPR}_{0}^{\mathrm{H}_{2}}=1$.

The fiducial choice of $n_{\mathrm{H}, 0} / n_{\mathrm{H}, 1}$, allows exploration over an order of magnitude change in density, starting from values of $\sim$ few $\times 10^{4} \mathrm{~cm}^{-3}$. These initial conditions still correspond to relatively dense regions of molecular clouds, i.e., typical IRDC conditions. Here we expect there to already be moderate depletion of $\mathrm{CO}$, with $f_{D} \simeq 3$ observed by Hernandez et al. (2011, 2012), which thus motivates the choice of fiducial value. We will also explore models with $n_{\mathrm{H}, 0} / n_{\mathrm{H}, 1}=0.01$ and $f_{D, 0}=1$ and 10 . The initial $\mathrm{OPR}^{\mathrm{H}_{2}}$ ratio is quite uncertain. Crabtree et al. (2011) measured $\mathrm{OPR}^{\mathrm{H}_{2}} \sim 0.3-0.8$ in diffuse molecular clouds. We choose $\mathrm{OPR}_{0}^{\mathrm{H}_{2}}=1$ as a fiducial value, but also explore the effects of other, especially lower, values, across a range of $\mathrm{OPR}_{0}^{\mathrm{H}_{2}}=0.01-3$. Other parameters of the modeling are the same as those listed in Tables 1 and 2 of K15.

Figures 6 and 7 show the results of the astrochemical modeling of C1-N and C1-S with $f_{D, 0}=1,3,10$, and including the different rates of density evolution as the core contracts with $\alpha_{\mathrm{ff}}=0.01,0.033,0.1,0.33,1$. The corresponding evolution of $\left[\mathrm{N}_{2} \mathrm{D}^{+}\right], D_{\text {frac }}^{\mathrm{N}_{2} \mathrm{H}^{+}}$, and $\left[\mathrm{o}-\mathrm{H}_{2} \mathrm{D}^{+}\right]$are also shown. Here square parentheses denote fractional abundance relative to total $\mathrm{H}$ nuclei number density. The Case 1 and Case 2 observational constraints for $\left[\mathrm{N}_{2} \mathrm{D}^{+}\right]$and $D_{\text {frac }}^{\mathrm{N}_{2} \mathrm{H}^{+}}$, plus the limits on $\left[\mathrm{o}-\mathrm{H}_{2} \mathrm{D}^{+}\right]$are indicated with the shaded red regions (additional systematic uncertainties in $\left[\mathrm{N}_{2} \mathrm{D}^{+}\right]$, due to factor of approximately two uncertainties in $N_{\mathrm{H}}$, are shown with a lighter shade; the extremes of the Case 1 and 2 estimates for $D_{\text {frac }}^{\mathrm{N}_{2} \mathrm{H}^{+}}$define the shaded region; the effect of the $T_{\text {ex }}$ uncertainty from 4 to $7 \mathrm{~K}$ on the upper limit of $\left[\mathrm{o}-\mathrm{H}_{2} \mathrm{D}^{+}\right]$is also shown with a lighter shade).

Note that there are also potential systematic uncertainties associated with the theoretical astrochemical modeling, which for the abundances and abundance ratios of interest are at approximately the factor of two level (K15), e.g., as evidenced by the systematic differences of the results of our chemical network compared to that of Sipilä et al. (2015).

Considering the fiducial $f_{D, 0}=3$ case for C1-N and C1-S, the primary effect to note is that in rapidly collapsing cores, i.e., $\alpha_{\mathrm{ff}} \sim 1$, there is too little time for the level of deuteration to rise to very high values, so the core exhibits $D_{\text {frac }}^{\mathrm{N}_{2} \mathrm{H}^{+}} \sim 10^{-3}$. For more slowly evolving cores with $\alpha_{\mathrm{ff}} \lesssim 0.3$, there is time for the core to reach near equilbrium values of $\left[\mathrm{N}_{2} \mathrm{D}^{+}\right]$and $D_{\text {frac }}^{\mathrm{N}_{2} \mathrm{H}^{+}} \sim 0.1-1$.

Figures 8 and 9 show summaries of the modeling results of $\mathrm{C} 1-\mathrm{N}$ and $\mathrm{C} 1-\mathrm{S}$, respectively. The three-dimensional parameter space of $\left[\mathrm{N}_{2} \mathrm{D}^{+}\right], D_{\text {frac }}^{\mathrm{N}_{2} \mathrm{H}^{+}}$, and $\left[0-\mathrm{H}_{2} \mathrm{D}^{+}\right]$is shown for each of the cases with $f_{D, 0}=1,3,10$. The locations of the models at $t=t_{1}$ (i.e., present-day core conditions) are shown with the colored square points with factor of two theoretical uncertainties indicated. Observational constraints on $\left[\mathrm{N}_{2} \mathrm{D}^{+}\right], D_{\text {frac }}^{\mathrm{N}_{2} \mathrm{H}^{+}}$, and [o$\mathrm{H}_{2} \mathrm{D}^{+}$] are again depicted by the shaded red regions as described above.

In principle, in each panel of Figures 8 and 9, models that fall into the overlapping red areas are the ones consistent with all of the observational constraints, although leeway should be given for potential theoretical model uncertainties. The $D_{\text {frac }}^{\mathrm{N}_{2} \mathrm{H}^{+}}$ constraints are the most stringent and the high observed values of $D_{\text {frac }}^{\mathrm{N}_{2} \mathrm{H}^{+}} \gtrsim 0.1$ for both C1-N and C1-S allow us to rule out the fastest collapsing $\alpha_{\mathrm{ff}}=1$ models, regardless of the initial depletion factor (when the core was at a 10 times smaller density). Models with $\alpha_{\mathrm{ff}}=0.01,0.033,0.1,0.33$ give a much better match to the observational contraints. In fact, the observational estimates are broadly consistent with the chemical equilbrium values, which the slow-collapsing models have time to converge to.

In C1-S, some of the slower collapsing models begin to predict abundances of $\mathrm{N}_{2} \mathrm{D}^{+}$that are moderately higher than the observational constraints, with the slowest collapsing models with $\alpha_{\mathrm{ff}}=0.01$ having the smallest discrepancies. However, these differences are relatively small (a factor of a few), compared to the difficulties faced by the $\alpha_{\mathrm{ff}}=1$ models.

\subsection{Parameter Space Exploration}

Here we explore the effects of varying model parameters, including initial ortho-to-para ratio of $\mathrm{H}_{2}\left(\mathrm{OPR}_{0}^{\mathrm{H}_{2}}\right)$, cosmic-ray ionization rate $(\zeta)$, and initial density relative to final density $\left(\delta n_{\mathrm{H}}^{\prime} \equiv n_{\mathrm{H}, 0} / n_{\mathrm{H}, 1}\right)$. Based on the results of K15, temperature variation does not have a significant impact to deuterium chemistry at $T_{k} \lesssim 15 \mathrm{~K}$. Since the temperatures in $\mathrm{C} 1-\mathrm{N}$ and C1-S are $\lesssim 13 \mathrm{~K}$ (T13), we keep the fiducial value of $T_{k}=10 \mathrm{~K}$.

Figures 10 and 11 show exploration with the higher cosmicray ionization rate $\zeta=10^{-16} \mathrm{~s}^{-1}$ (four times higher than the fiducial value). Compared to the fiducial models (Figures 8 and 9), there are two notable changes. First, the fast collapsing models $\left(\alpha_{\mathrm{ff}}=0.33,1.0\right)$ have higher $D_{\mathrm{frac}}^{\mathrm{N}_{2} \mathrm{H}^{+}}$. In particular, the $\alpha_{\mathrm{ff}}=0.33$ model reaches the observed $D_{\mathrm{frac}}^{\mathrm{N}_{2} \mathrm{H}^{+}}$, even if there is no initial depletion. This higher rate of increase of deuteration is a direct consequence of the higher value of $\zeta$. Consequently, $D_{\text {frac }}^{\mathrm{N}_{2} \mathrm{H}^{+}}$in the fast collapsing models is closer to the equilibrium value. A second change is a decrease in $D_{\mathrm{frac}}^{\mathrm{N}_{2} \mathrm{H}^{+}}$of the slower collapsing models, e.g., $\alpha_{\mathrm{ff}}=0.03,0.01$. This is also expected from the results of K15, where high $\zeta$ reduces the equilibrium 


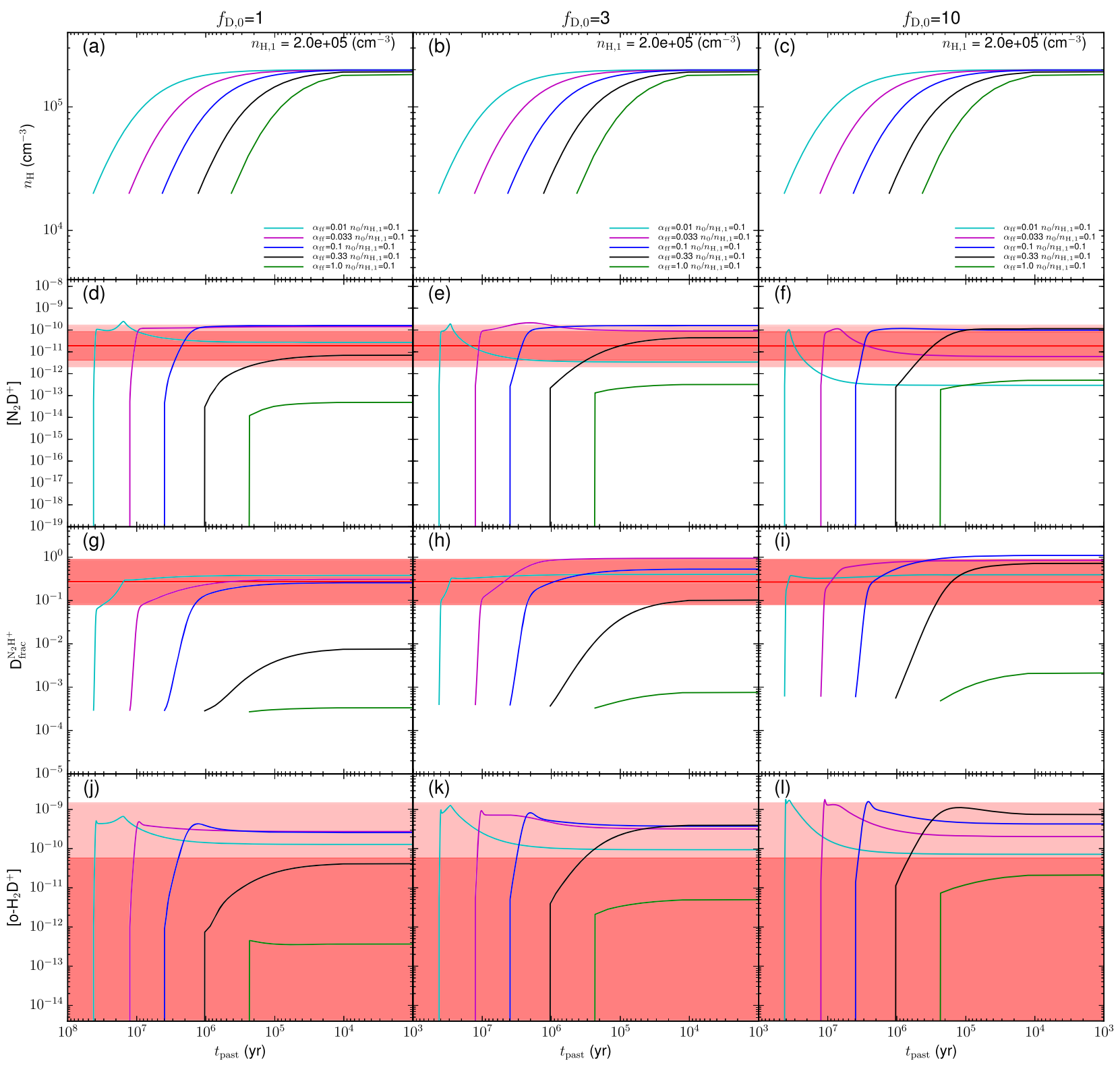

Figure 6. Chemodynamical modeling of C1-N. The models include time-dependent depletion/desorption (TDD) of heavy elements onto dust grains and dynamical density evolution (DDE), as parameterized by $\alpha_{\mathrm{ff}}$ (see Equation (4)). For C1-N, the models have target, present-day density $n_{\mathrm{H}, 1}=2.05 \times 10^{5} \mathrm{~cm}^{-3}$. The columns from left to right show results for initial heavy element depletion factors of $f_{D, 0}=1,3$ (fiducial), 10. Top row: time evolution of density as a function of $t_{\text {past }}$, which increases to the left. Models with $\alpha_{\mathrm{ff}}=0.01,0.033,0.1,0.33,1$ and starting to final density ratios of $n_{\mathrm{H}, 0} / n_{\mathrm{H}, 1}=0.1$ are shown. Second row: time evolution of $\left[\mathrm{N}_{2} \mathrm{D}^{+}\right]$for these various models. Case 1 and 2 observational estimates for $\left[\mathrm{N}_{2} \mathrm{D}^{+}\right]$set the darker shaded region, with additional systematic uncertainties due to factor of approximately two uncertainties in $N_{\mathrm{H}}$ shown with a lighter shade. Third row: time evolution of $D_{\text {frac }}^{\mathrm{N}_{2} \mathrm{H}^{+}}$for the same models. The extremes of the Case 1 and 2 estimates for $D_{\text {frac }}^{\mathrm{N}_{2} \mathrm{H}^{+}}$set the range of the shaded region. Note that the fast collapsing models do not have enough time to reach large abundances of $\mathrm{N}_{2} \mathrm{D}^{+}$or large values of $D_{\text {frac }}^{\mathrm{N}_{2} \mathrm{H}^{+}}$. (d) Bottom row: time evolution of $\left[\mathrm{o}-\mathrm{H}_{2} \mathrm{D}^{+}\right]$for the same models. The observational upper limit on [o- $\left.\mathrm{H}_{2} \mathrm{D}^{+}\right]$is shown with the red shaded region, with the effect of the $T_{\mathrm{ex}}$ uncertainty from 4 to $7 \mathrm{~K}$ shown with a lighter shade (this dominates over the effect of $N_{\mathrm{H}}$ uncertainties).

$D_{\text {frac }}^{\mathrm{N}_{2} \mathrm{H}^{+}}$. The slow-collapsing models have more than enough time to reach this equilbrium value.

Figures 12 and 13 show the effect of varying the ratio of the initial model density compared to the final density. The models here have $n_{\mathrm{H}, 0}=0.01 n_{\mathrm{H}, 1}$ (while the fiducial case assumed $\left.n_{\mathrm{H}, 0}=0.1 n_{\mathrm{H}, 1}\right)$. The main effect of starting with a lower density is that there is more time for chemical evolution of the gas so that deuteration equilibrium can be reached in faster collapsing models.

Figures 14 and 15 show another variation from the fiducial case, with $\mathrm{OPR}_{0}^{\mathrm{H}_{2}}=0.1$ (compared to $\mathrm{OPR}_{0}^{\mathrm{H}_{2}}=1$ in the fiducial models). In general, high $\mathrm{OPR}^{\mathrm{H}_{2}}$ suppresses deuteration. $D_{\text {frac }}^{\mathrm{N}_{2} \mathrm{H}^{+}}$does not reach equilibrium until the ortho-to-para ratio of $\mathrm{H}_{2}$ has dropped significantly. The typical timescale for ortho-to-para $\mathrm{H}_{2}$ conversion is of the order of one million years, depending on physical conditions, and so is the deuterium fractionation timescale. If models start with lower $\mathrm{OPR}_{0}^{\mathrm{H}_{2}}$, then the establishment of $\mathrm{OPR}^{\mathrm{H}_{2}}$ equilibrium is quicker. So the main difference between the models shown here and the fiducial models are that fast collapsing models can reach higher $D_{\mathrm{frac}}^{\mathrm{N}_{2} \mathrm{H}^{+}}$. This helps make the $\alpha_{\mathrm{ff}}=0.3$ model more consistent with the observations in terms of $D_{\text {frac }}^{\mathrm{N}_{2} \mathrm{H}^{+}}$. 


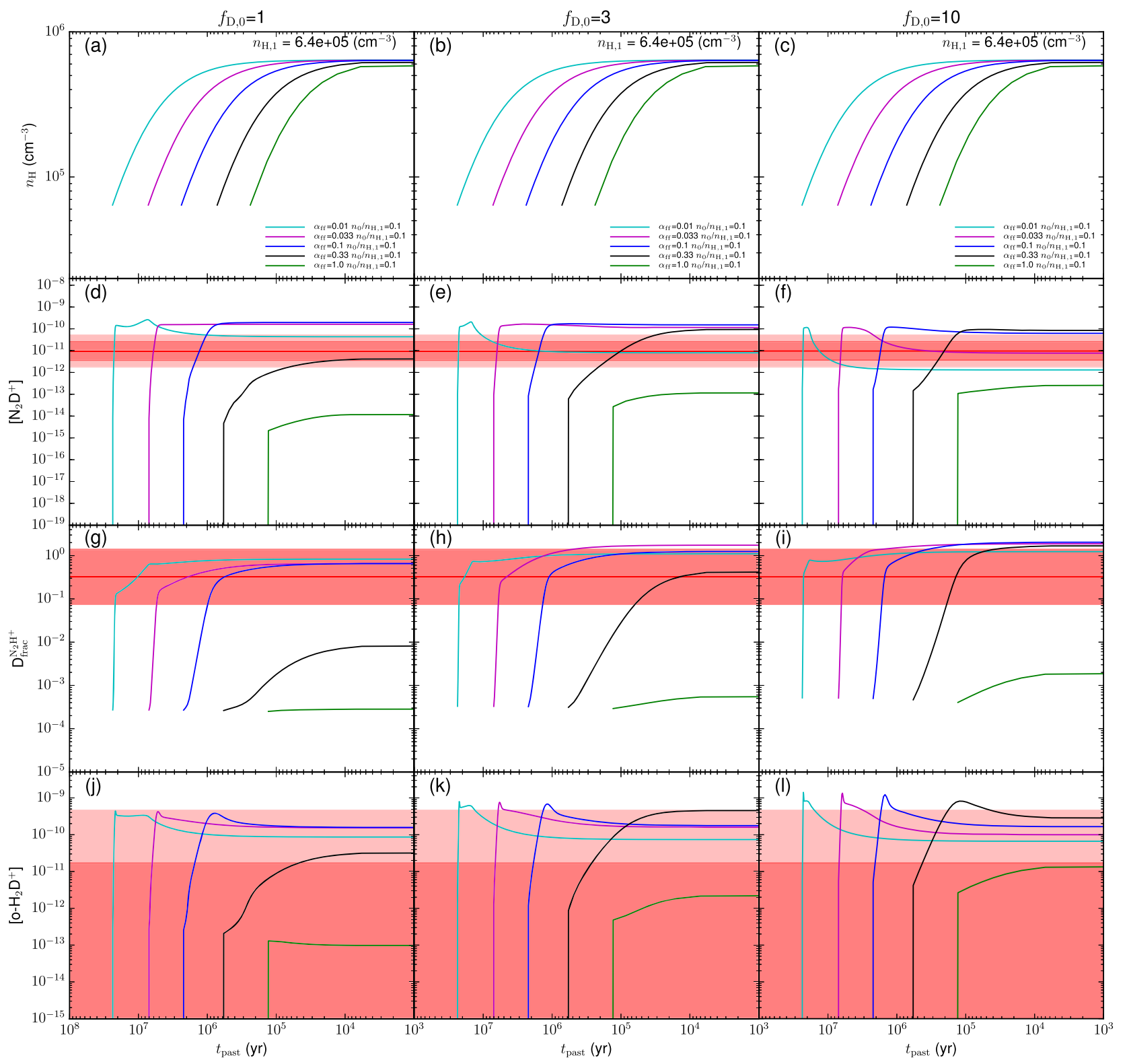

Figure 7. Same as Figure 6, but now for C1-S with a target, present-day density of $n_{\mathrm{H}, 1}=6.43 \times 10^{5} \mathrm{~cm}^{-3}$.

However, it is also important to note that by lowering the $\mathrm{OPR}_{0}^{\mathrm{H}_{2}}$ to be 0.1 for the initial condition, we are in effect starting with a chemically evolved and therefore relatively old molecular cloud as the initial condition for dense gas core formation.

\subsection{Best-fit $\alpha_{f f}$}

For each $\alpha_{\mathrm{ff}}$, we explore the other model parameters, compare with observational constraints and combine the results to estimate a likelihood parameter. The explored parameters are cosmic-ray ionization rate $\zeta=1.0 \times 10^{-18} \mathrm{~s}^{-1}$, $3.3 \times 10^{-18} \mathrm{~s}^{-1}, \quad 1.0 \times 10^{-17} \mathrm{~s}^{-1}, \quad 3.3 \times 10^{-17} \mathrm{~s}^{-1}$, $1.0 \times 10^{-16} \mathrm{~s}^{-1}$ (extension to lower values allows for the possibility of magnetic mirror shielding and attenuation of cosmic rays in dense, magnetized cloud cores Padovani \& Galli 2011), initial density relative to final density $\delta n_{\mathrm{H}}^{\prime} \equiv$ $n_{\mathrm{H}, 0} / n_{\mathrm{H}, 1}=0.1,0.01$, initial depletion factor $f_{D, 0}=1,3,10$, and initial ortho-to-para $\mathrm{H}_{2}$ ratio $\mathrm{OPR}_{0}^{\mathrm{H}_{2}}=3,1,0.1,0.01$. For each specific model $\left[\alpha_{\mathrm{ff}}, \zeta, \delta n_{\mathrm{H}}^{\prime}, f_{D, 0}, \mathrm{OPR}_{0}^{\mathrm{H}_{2}}\right]$, we calculate its total (summed in quadrature) "distance," $\Delta$, in the threedimensional log-scale parameter space to the "observed location" of $\left[\mathrm{N}_{2} \mathrm{D}^{+}\right], D_{\text {frac }}^{\mathrm{N}_{2} \mathrm{H}^{+}}$, [o- $\left.\mathrm{H}_{2} \mathrm{D}^{+}\right]$normalized by the $\log$ spacewidth of the observational constraint. The observed location for $\left[\mathrm{N}_{2} \mathrm{D}^{+}\right]$and $D_{\mathrm{frac}}^{\mathrm{N}_{2} \mathrm{H}^{+}}$is defined as the geometric mean value of the upper and lower limits (combining Cases 1 and 2). If the model result is between the lower and upper limits, its contribution to the total distance is set to zero. For [o$\mathrm{H}_{2} \mathrm{D}^{+}$], the observed location is set at the upper limit resulting from $T_{\mathrm{ex}}=7 \mathrm{~K}$. If the model value is below $\left[0-\mathrm{H}_{2} \mathrm{D}^{+}\right]$at $T_{\mathrm{ex}}=7 \mathrm{~K}$, the distance contribution is zero. Otherwise, it is the log-space difference (to the $7 \mathrm{~K}$ location) normalized by the distance from the $7 \mathrm{~K}$ location to the upper limit. Note that we also allow for a potential factor of two systematic uncertainty in the abundances $\left[\mathrm{N}_{2} \mathrm{D}^{+}\right]$and $\left[\mathrm{o}-\mathrm{H}_{2} \mathrm{D}^{+}\right]$(to either higher or lower values) due to the uncertainty in the observed $\mathrm{H}$ column density. 


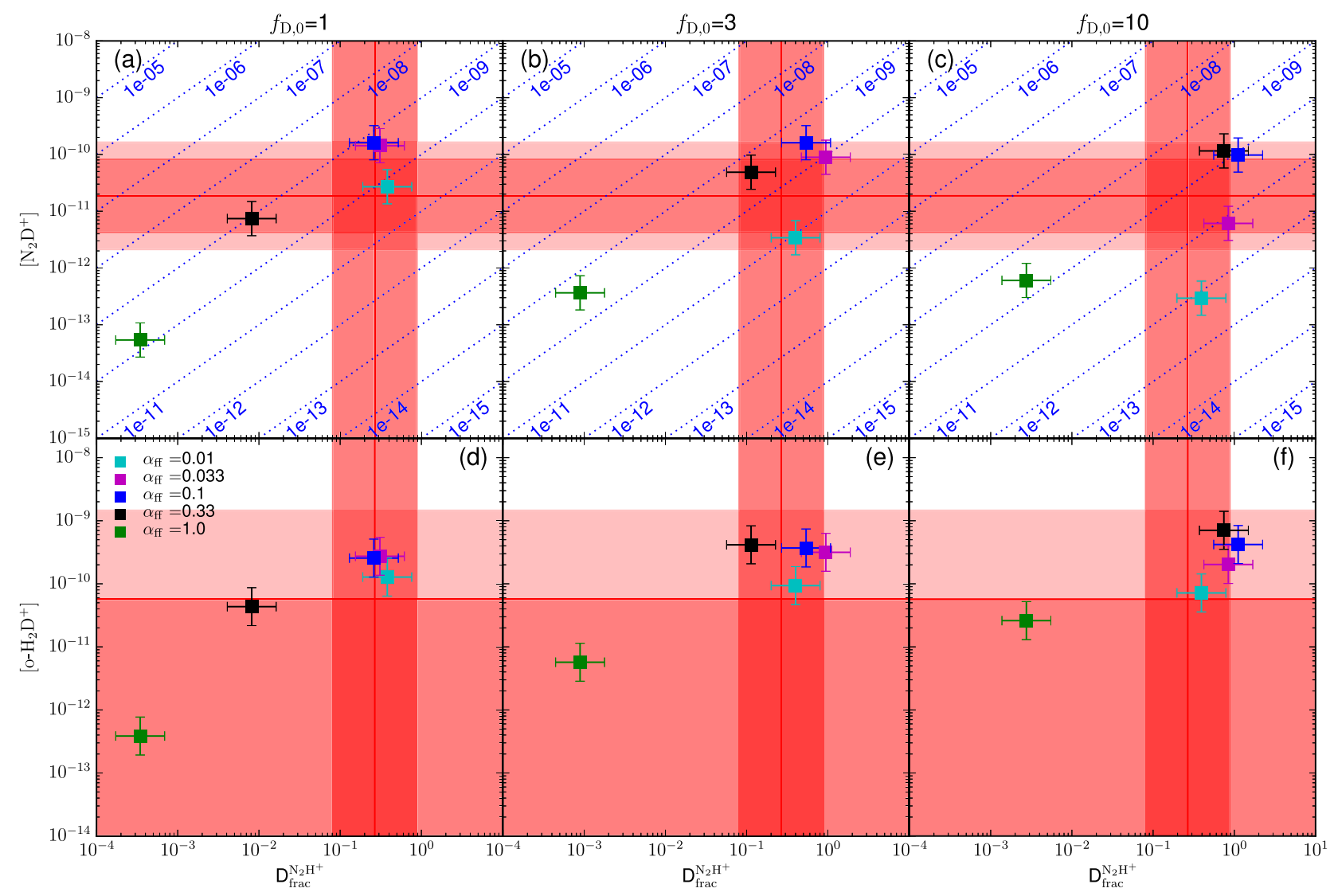

Figure 8. C1-N fiducial models and observational constraints in the $\left[\mathrm{N}_{2} \mathrm{D}^{+}\right]-D_{\text {frac }}^{\mathrm{N}_{2} \mathrm{H}^{+}}$(top row) and $\left[\mathrm{o}-\mathrm{H}_{2} \mathrm{D}^{+}\right]-D_{\text {frac }}^{\mathrm{N}_{2} \mathrm{H}^{+}}$(bottom row) parameter space. The blue dotted lines in the top row show constant $\left[\mathrm{N}_{2} \mathrm{H}^{+}\right]$values. The three columns show different initial depletion factors, $f_{D, 0}=1,3,10$, for the fiducial astrochemical models, the results of which are indicated by the squares, with different colors representing different collapse rate parameter values $\left(\alpha_{\mathrm{ff}}=0.01-1\right.$; see the legend). Factor of two systematic theoretical errors are indicated by the error bars around each point. All models are evolved to the final, observed density starting from a 10 times lower density, and the initial ortho-to-para ratio of $\mathrm{H}_{2}$ is set to one in all of these cases. The red shaded areas show the same observational constraints as described in Figure 6. Fast collapsing $\alpha_{\mathrm{ff}}=1$ models are not able to reach the large observed values of $D_{\mathrm{frac}}^{\mathrm{N}_{2} \mathrm{H}^{+}}$(see the text).

Then, considering the two values of $\delta n_{\mathrm{H}}^{\prime}=0.01,0.1$ separately, for each $\alpha_{\mathrm{ff}}$, we average the total distances from each specific model $\left[\zeta, f_{D, 0}, \mathrm{OPR}_{0}^{\mathrm{H}_{2}}\right]$ to have a likelihood parameter $\bar{\Delta}\left(\alpha_{\mathrm{ff}}, \delta n_{\mathrm{H}}\right)$. Lower $\bar{\Delta}$ means better agreement. The results are listed in Table 4. Note that, for this simple test, we do not give special weighting to any of the parameters, i.e., we assume that all parameters are equally important (which has guided the range of parameters considered).

For both C1-N and C1-S, the best-fitting values of $\alpha_{\mathrm{ff}}$ are $\ll 1$, which would suggest that both $\mathrm{C} 1-\mathrm{N}$ and $\mathrm{C} 1-\mathrm{S}$ are contracting very slowly compared to free-fall collapse. However, the more meaningful constraint is that $\alpha_{\mathrm{ff}} \gtrsim 0.3$ models are disfavored with their values of $\bar{\Delta}$ greater than the best-fit models by $\gtrsim 50 \%$.

Figures 16 and 17 show some of the most promising models that satisfy the observational constraints for C1-N with $\delta n_{\mathrm{H}}^{\prime}=0.1$ and 0.01 , respectively. Figures 18 and 19 show the equivalent models for C1-S. For C1-S, we have included models that fall within a factor of two of the observational constraints, which allows for theoretical uncertainties. These figures also show time evolution of abundances $\left[\mathrm{DCO}^{+}\right]$and [DCN], which with future observational constraints may help to discriminate between the models.

For C1-S with $\delta n_{\mathrm{H}}^{\prime}=0.1$ and focussing on models with $f_{D, 0} \geqslant 3$, we see that the majority of models have $\alpha_{\mathrm{ff}} \leqslant 0.33$. Fast collapse models with $\alpha_{\mathrm{ff}}=1$ require either relatively low values of $\mathrm{OPR}^{\mathrm{H}_{2}}$ (which would imply an already chemical evolved initial condition) or relatively high values of $\zeta$ or $f_{D, 0}$. Similar conclusions apply to the allowed C1-S models with $\delta n_{\mathrm{H}}^{\prime}=0.01$, including those starting with no initial depletion (which may be more reasonable for these lower initial densities). Improved observational constraints on $\left[\mathrm{N}_{2} \mathrm{D}^{+}\right]$, $D_{\text {frac }}^{\mathrm{N}_{2} \mathrm{H}^{+}}$, and $\left[\mathrm{o}-\mathrm{H}_{2} \mathrm{D}^{+}\right]$, along with new constraints on $\left[\mathrm{DCO}^{+}\right]$ and [DCN], will help to winnow out the allowed models.

For C1-N with $\delta n_{\mathrm{H}}^{\prime}=0.1$ and again focusing on models with $f_{D, 0} \geqslant 3$, we again see that most acceptable models require relatively small values of $\alpha_{\mathrm{ff}}$. With $\delta n_{\mathrm{H}}^{\prime}=0.01$, a small fraction of fast $\alpha_{\mathrm{ff}}=1$ models are allowed, but these again require low values of $\mathrm{OPR}^{\mathrm{H}_{2}}$, i.e., a chemically "aged" initial condition.

\section{DISCUSSION}

\subsection{Implications for Core Dynamics}

In order for the cores to be contracting slowly, $\left(\alpha_{\mathrm{ff}}<0.33\right)$, there should be a significant amount of support against gravity. T13 studied the dynamics of C1-N and C1-S. A virial analysis indicated that they are moderately sub-virial, unless magnetic fields of the order of $\sim 1 \mathrm{mG}$ are present. Our constraints from the deuteration chemical clock support this interpretation: a relatively long core history is needed, otherwise there would not be enough time to reach such high levels of deuteration $\left(D_{\text {frac }}^{\mathrm{N}_{2} \mathrm{H}^{+}} \gtrsim 0.1\right)$. Higher resolution observations that can begin to 


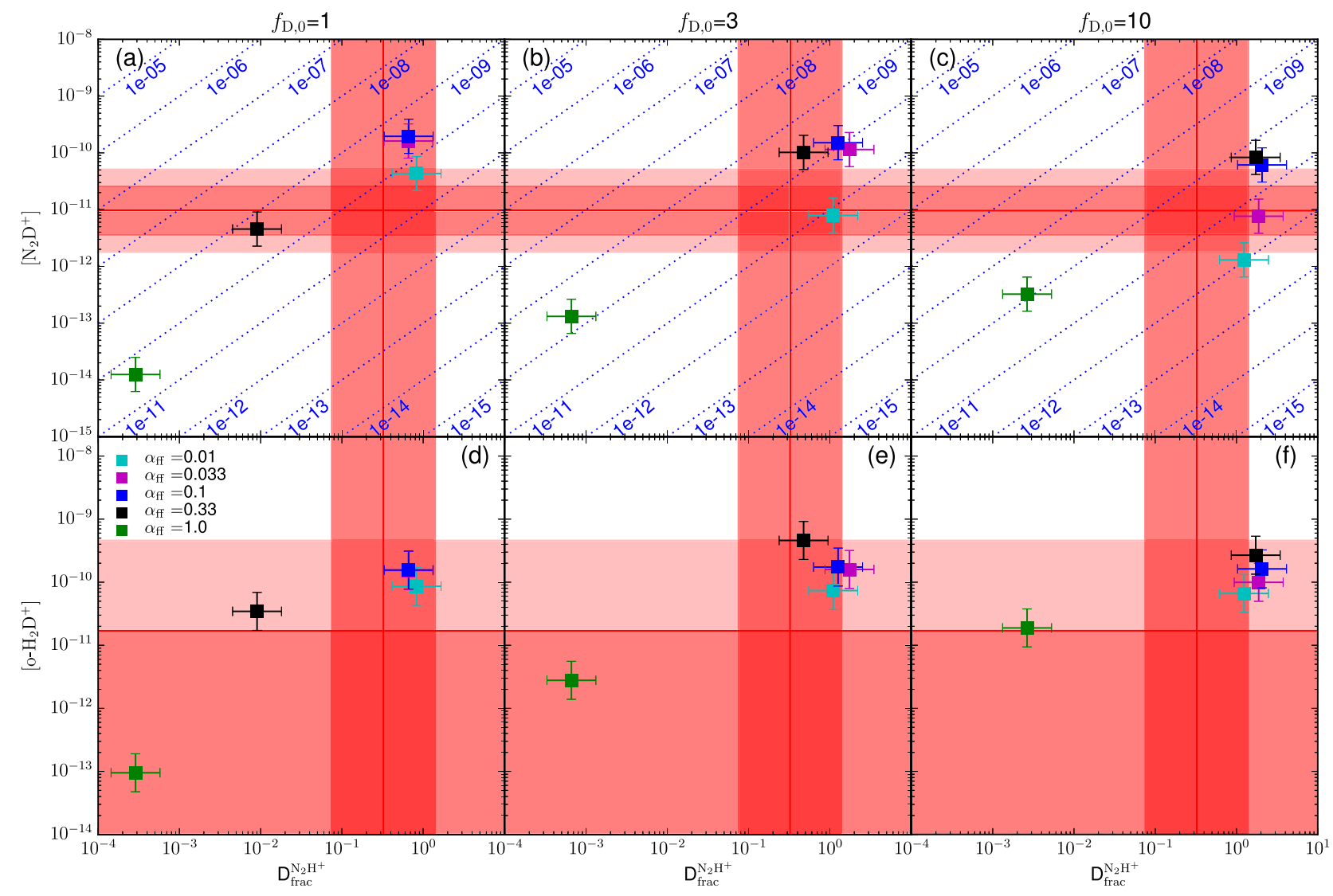

Figure 9. C1-S fiducial results, i.e., the same as Figure 8, but now for C1-S.

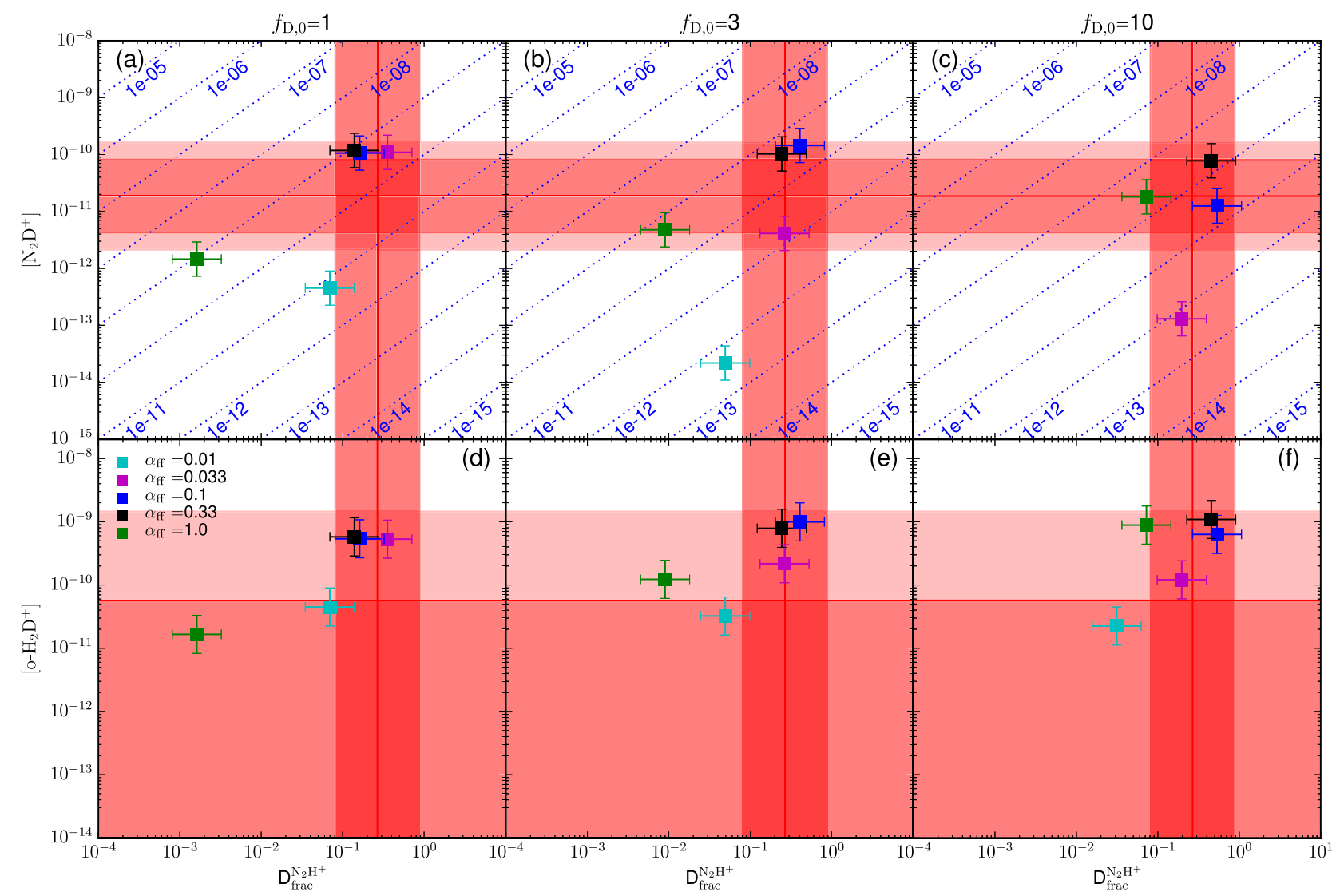

Figure 10. C1-N with high cosmic-ray ionization rate, i.e., the same as Figure 8, but now the astrochemical models are run with a higher cosmic-ray ionization rate $\zeta=10^{-16} \mathrm{~s}^{-1}$. 


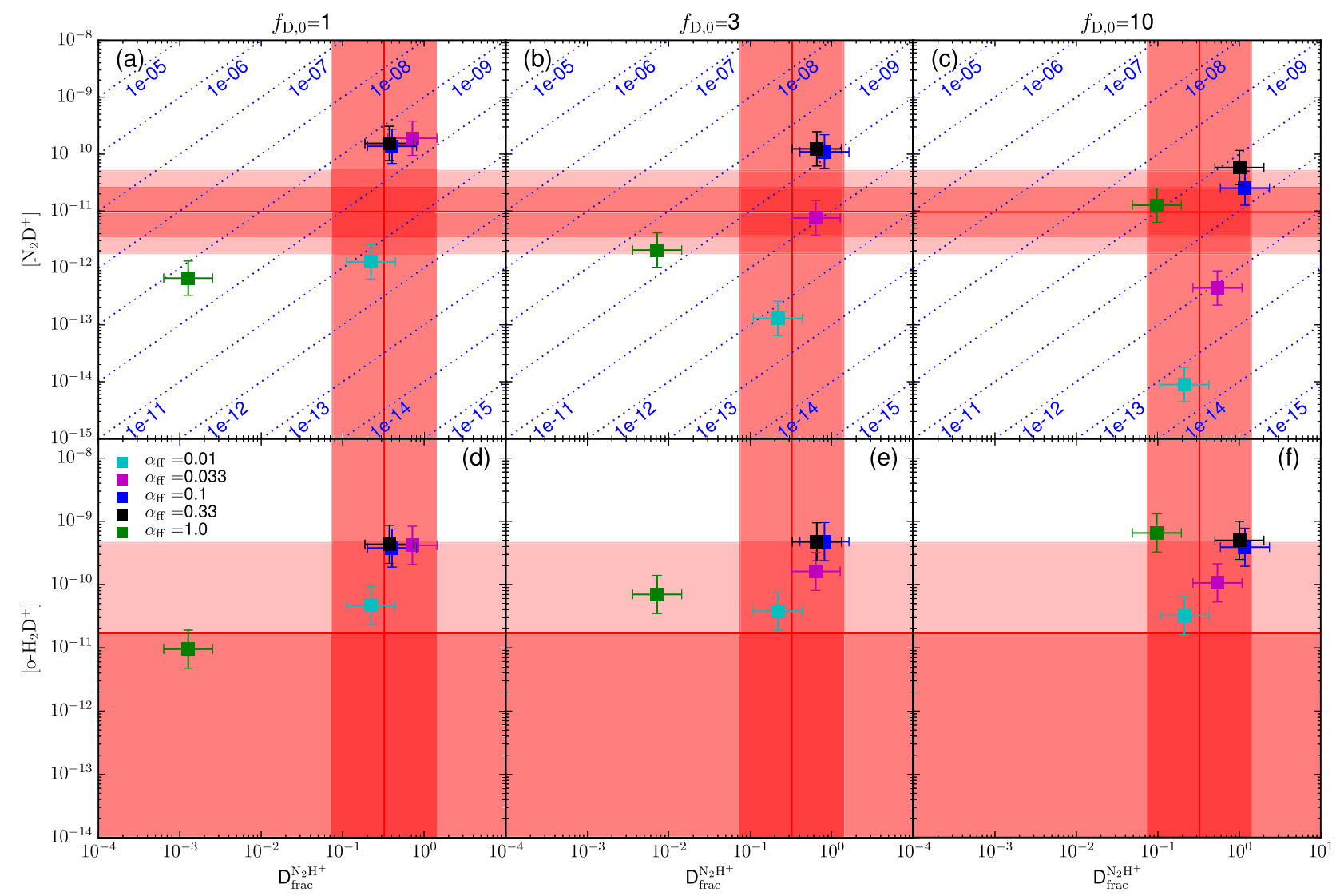

Figure 11. C1-S with high cosmic-ray ionization rate, i.e., the same as Figure 10, but now for C1-S.

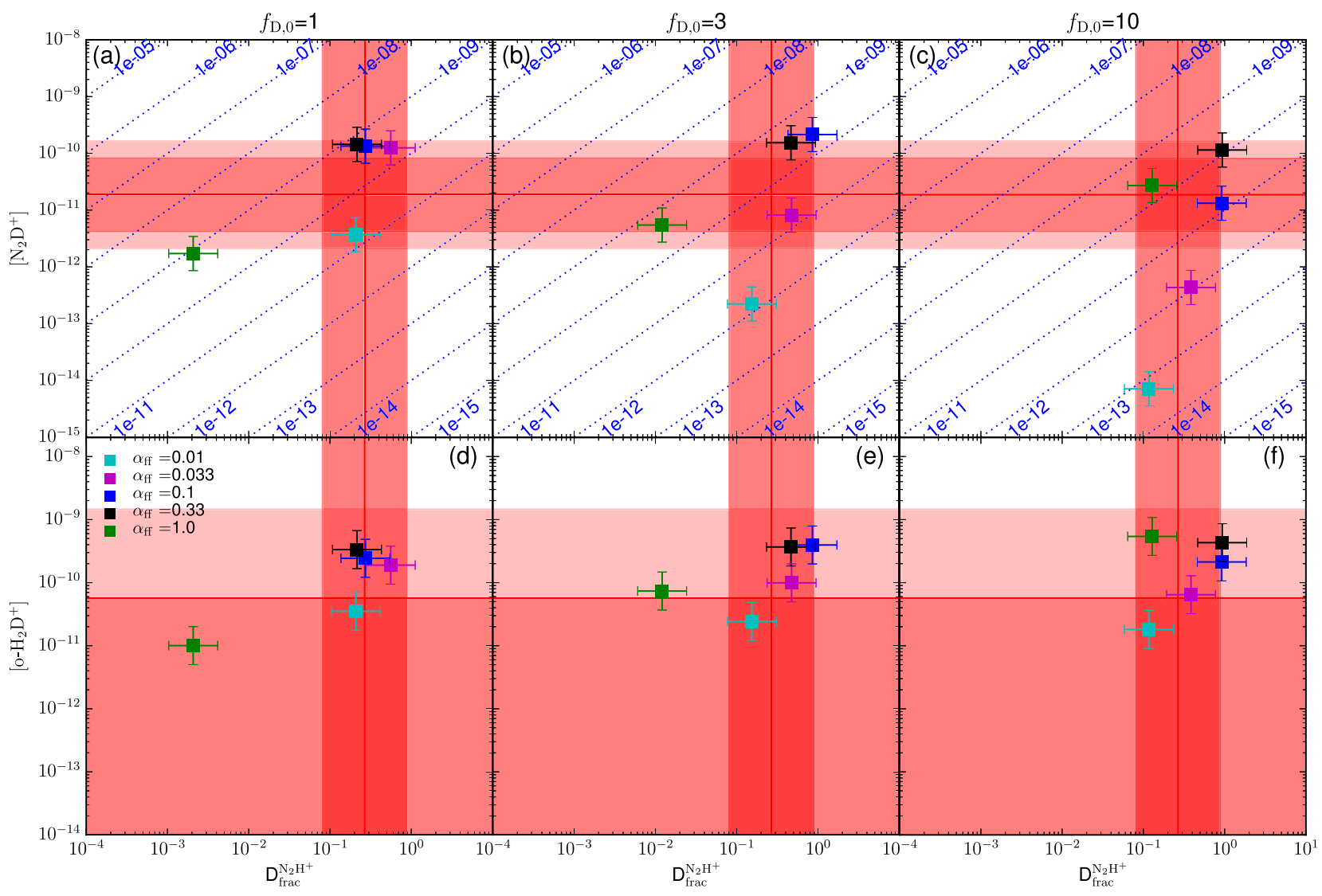

Figure 12. C1-N with low initial density, i.e., the same as Figure 8, but with $n_{\mathrm{H}, 0}=0.01 n_{\mathrm{H}, 1}$. 


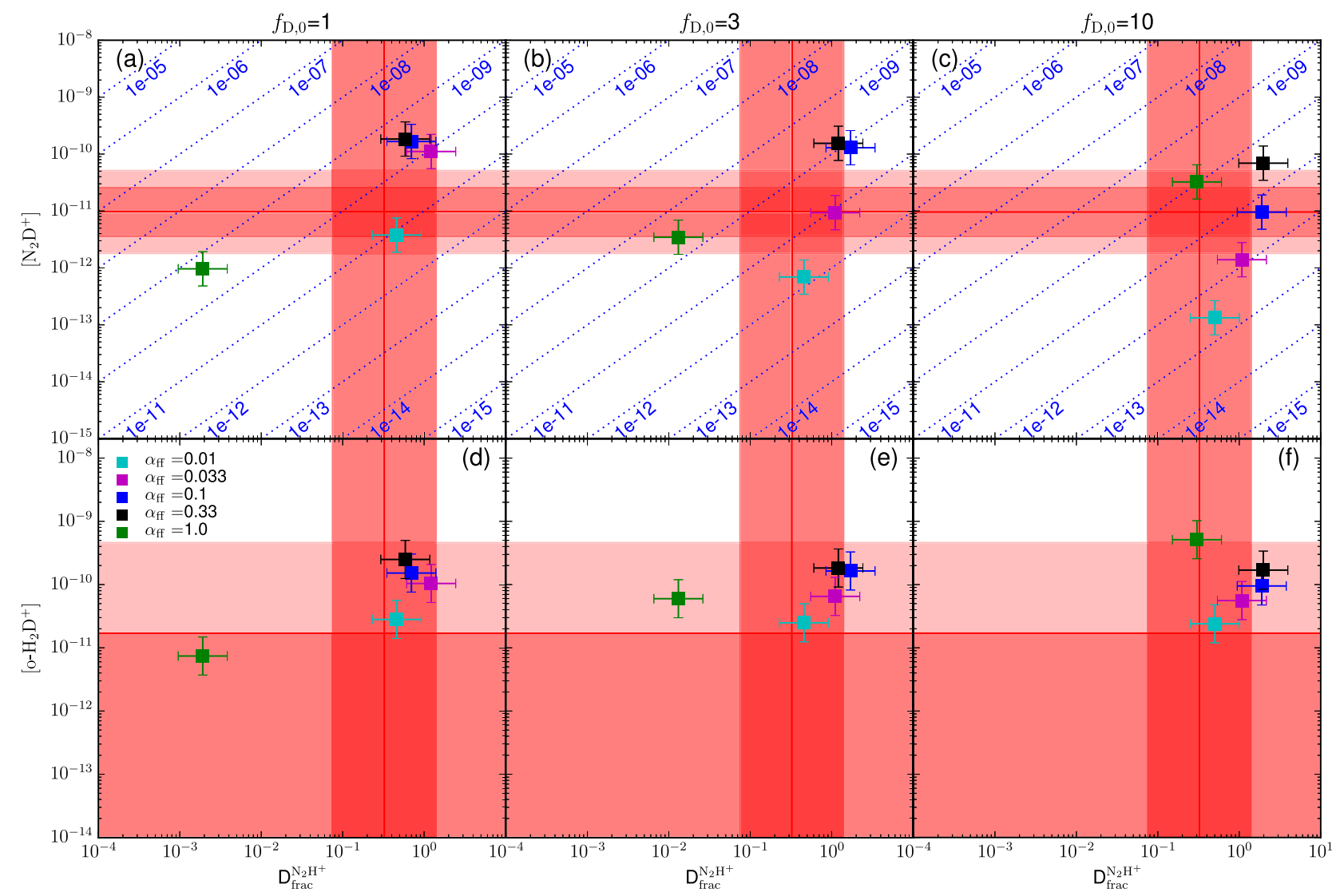

Figure 13. C1-S with low initial density, i.e., the same as Figure 12, but for C1-S.

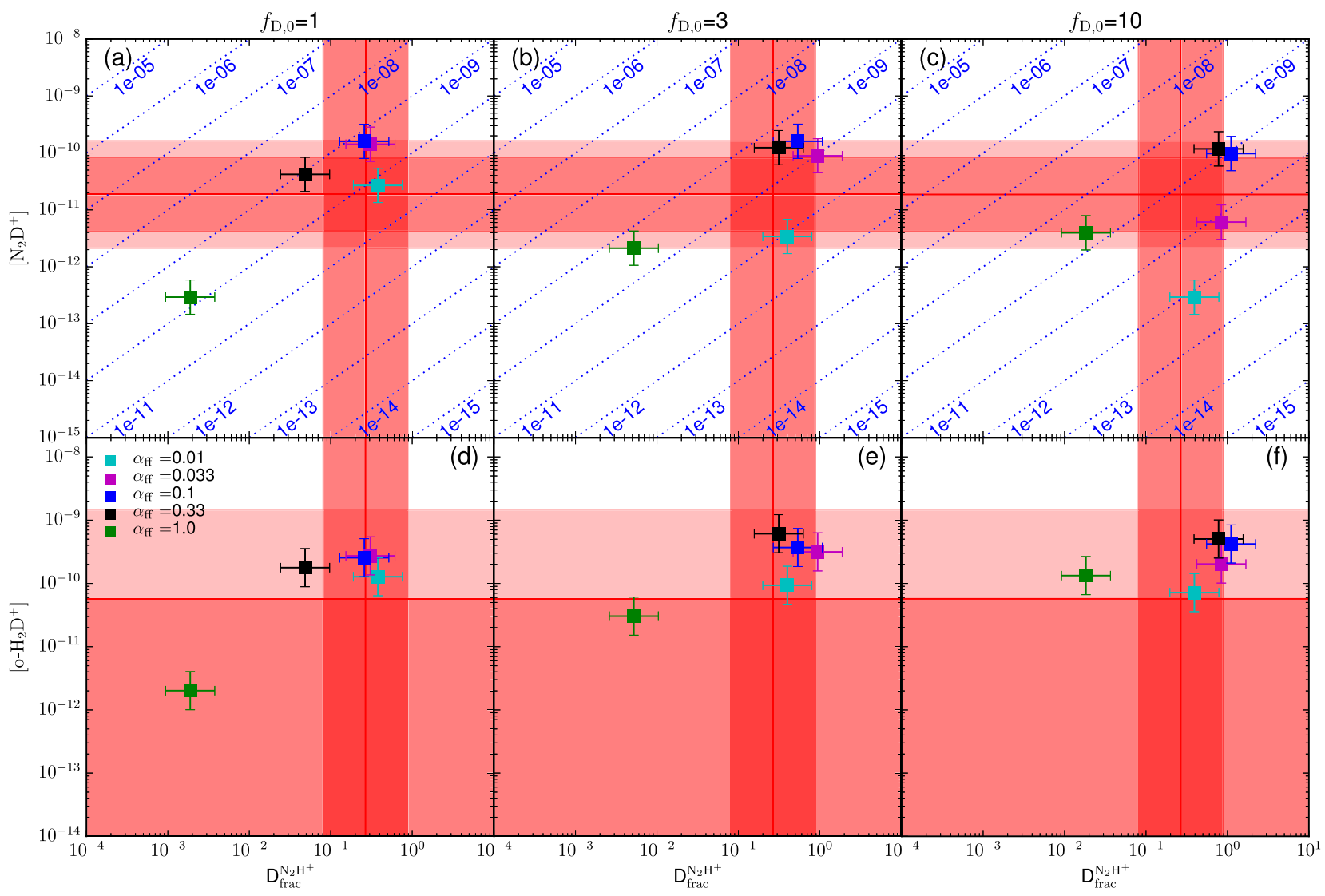

Figure 14. $\mathrm{C} 1-\mathrm{N}$ with low initial ortho-to-para ratio of $\mathrm{H}_{2}$, i.e., the same as Figure 8, but with $\mathrm{OPR}_{0}^{\mathrm{H}_{2}}=0.1$. 


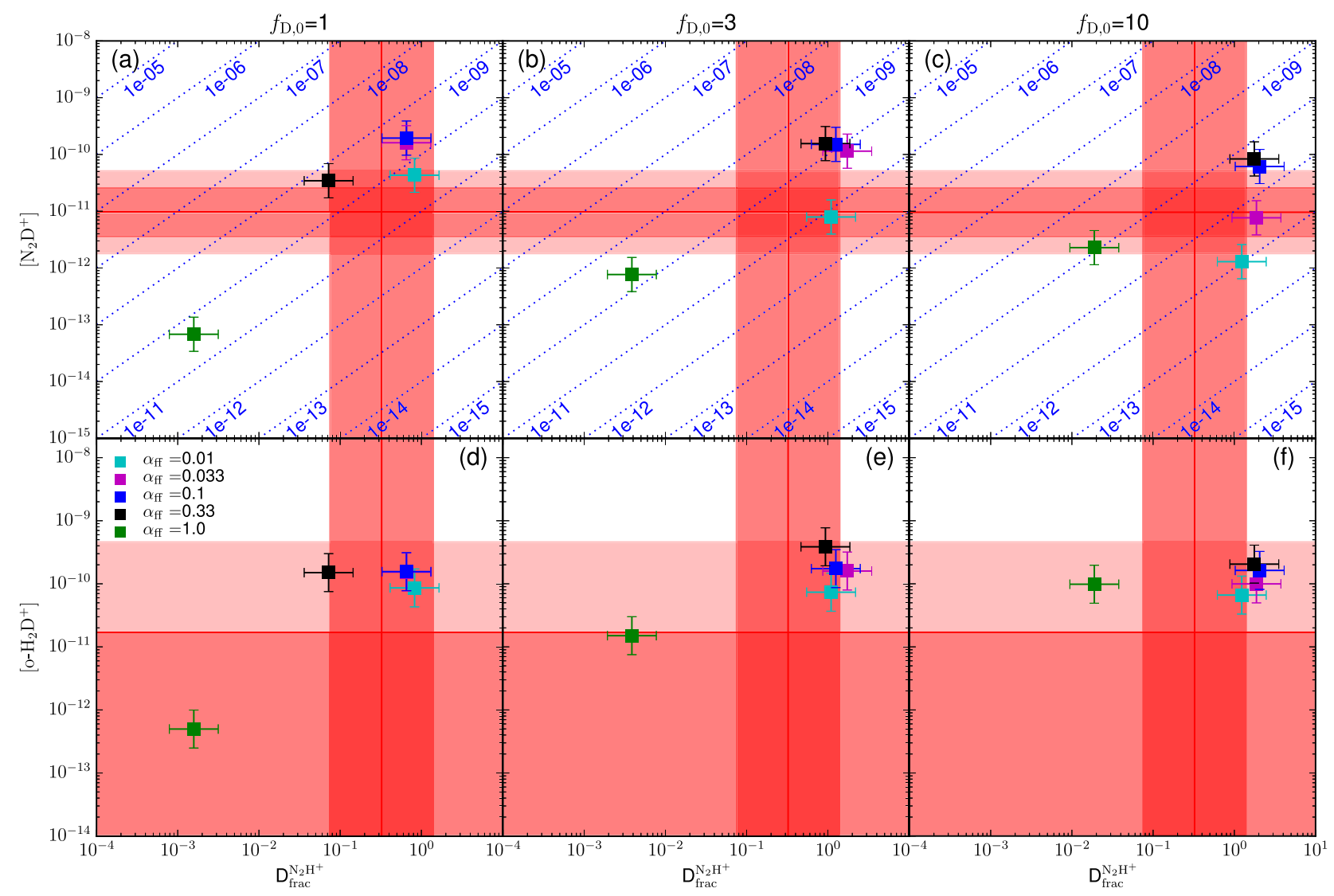

Figure 15. C1-S with low initial ortho-to-para ratio of $\mathrm{H}_{2}$, i.e., the same as Figure 14, but for C1-S.

Table 4

Likelihood Parameters, $\bar{\Delta}\left(\alpha_{\mathrm{ff}}, \delta n_{\mathrm{H}}^{\prime}\right)$, for Explored Astrochemical Models

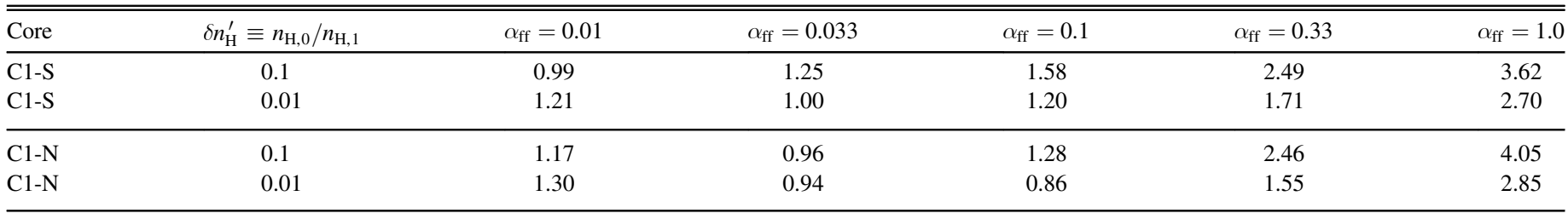

map $D_{\text {frac }}^{\mathrm{N}_{2} \mathrm{H}^{+}}$would provide stronger constraints, since relative $D_{\text {frac }}^{\mathrm{N}_{2} \mathrm{H}^{+}}$values would be more accurately measured and would constrain the properties of the envelope gas from which the cores are forming.

In terms of timescales of the contraction from $n_{\mathrm{H}, 0}$ to the present day, from Equation (4), we derive, e.g., for C1-S, $t_{\text {past }, 0}=(2.4,10.0) \times 10^{5} / \alpha_{\mathrm{ff}}$ year (for $\delta n_{\mathrm{H}}^{\prime}=0.1,0.01$, respectively). In the first case, the core age would be $t_{\text {past }, 0}=7.2 \times 10^{5}$ years with $\alpha_{\mathrm{ff}}=0.33$ and $2.4 \times 10^{6}$ years with $\alpha_{\mathrm{ff}}=0.1$. These timescales are several times longer than some estimates of low-mass starless core lifetimes in dense regions from statistics of starless cores, protostellar cores and young stellar objects (see review by Ward-Thompson et al. 2007, p. 33), but are comparable with the astrochemical estimate of the age of a protostellar core envelope by Brünken et al. (2014).

\section{2. $D_{\text {frac }}^{\mathrm{N}_{2} \mathrm{H}^{+}}$Measurement in Core}

Given the potential importance of $D_{\mathrm{frac}}^{\mathrm{N}_{2} \mathrm{H}^{+}}$as a useful chemical clock, the measurement of this quantity is worth more attention. Our initial goal of using multitransition spectral fitting was to obtain a more accurate measurement of $D_{\text {frac }}^{\mathrm{N}_{2} \mathrm{H}^{+}}$than simply using one $\mathrm{N}_{2} \mathrm{D}^{+}$line and one $\mathrm{N}_{2} \mathrm{H}^{+}$line. However, as in our case, this can bring more complexity, especially for $\mathrm{N}_{2} \mathrm{H}^{+}$that shows extended emission. The main sources of uncertainty come from the different spatial scales that are probed by the observations, with the single-dish observations not resolving the cores (and by varying amounts).

However, $\mathrm{N}_{2} \mathrm{D}^{+}$is less likely to suffer from this problem since it appears more spatially concentrated: i.e., the localized cores are in fact defined by their $\mathrm{N}_{2} \mathrm{D}^{+}(3-2)$ emission. Therefore, the $T_{\mathrm{ex}}$ derived from fitting simultaneously the $\mathrm{N}_{2} \mathrm{D}^{+}$lines should be more reliable.

Another potential difficulty is that while single-dish measurements gather the total flux in their beam, the interferometer data is only sensitive to structures with a specific range of sizes. However, we do not expect this to be a significant problem for at least our CARMA and ALMA data on the $\mathrm{C} 1-\mathrm{N} \& \mathrm{~S}$ cores. The angular size of both cores is $\sim 7^{\prime \prime}$. The angular resolution of ALMA observation is $2^{\prime \prime}$, and the maximum recoverable scale is $9^{\prime \prime}$. CARMA observation has $5^{\prime \prime}$ synthesized beam, and the maximum recoverable scale is $\sim 50^{\prime \prime}$. 


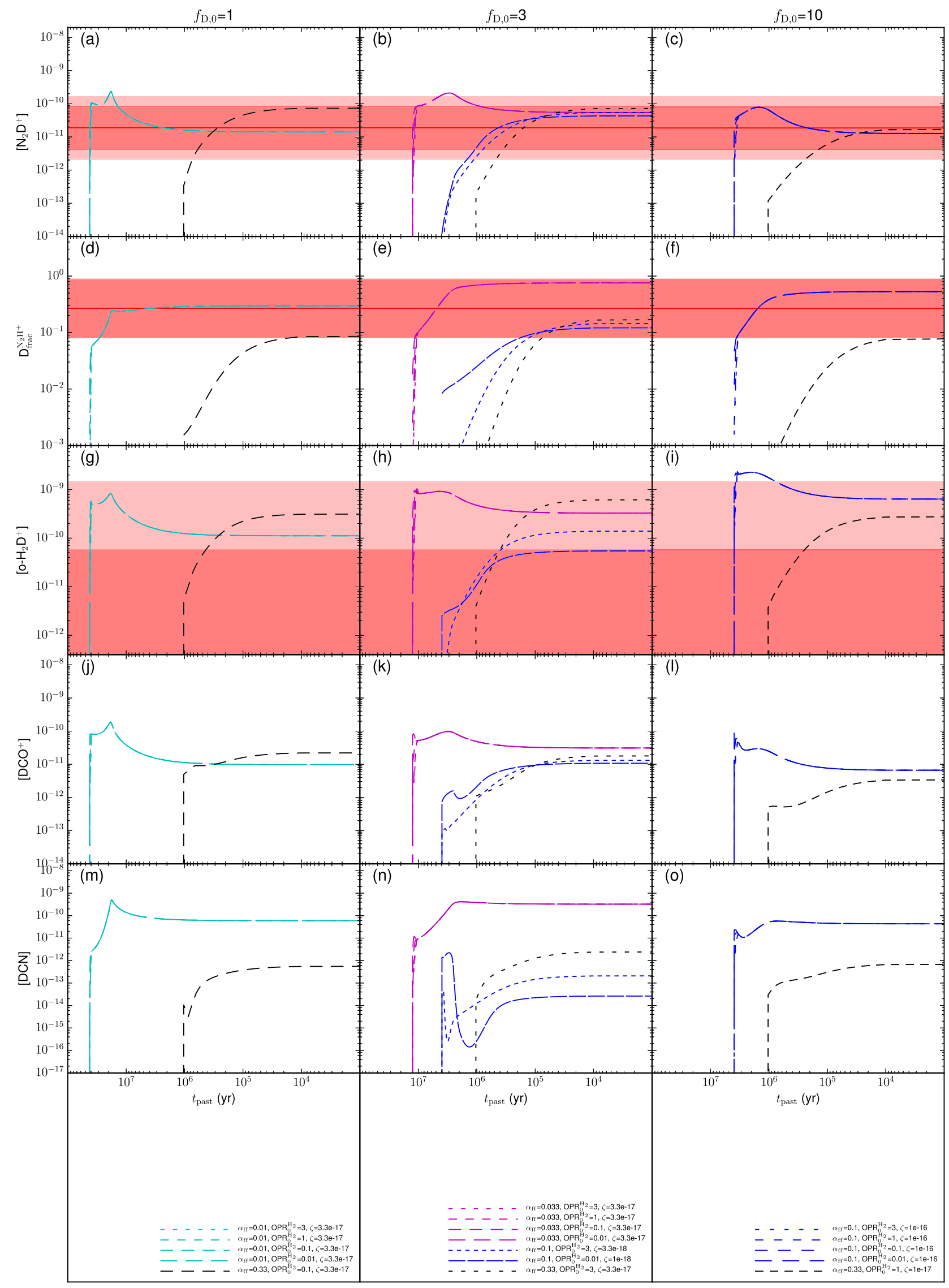

Figure 16. Most promising models for $\mathrm{C} 1-\mathrm{N}$ with $\delta n_{\mathrm{H}}^{\prime}=0.1$ from the parameter space exploration described in Section 4.2 , i.e., models that have final values of $\left[\mathrm{N}_{2} \mathrm{D}^{+}\right], D_{\text {frac }}^{\mathrm{N}_{2} \mathrm{H}^{+}}$, $\left[\mathrm{o}-\mathrm{H}_{2} \mathrm{D}^{+}\right]$within observational limits. The upper three rows follow the same format as the equivalent rows in Figure 6 . The next rows show time evolution of abundances $\left[\mathrm{DCO}^{+}\right]$and $[\mathrm{DCN}]$, which with future observational constraints may help to discriminate between the models. 


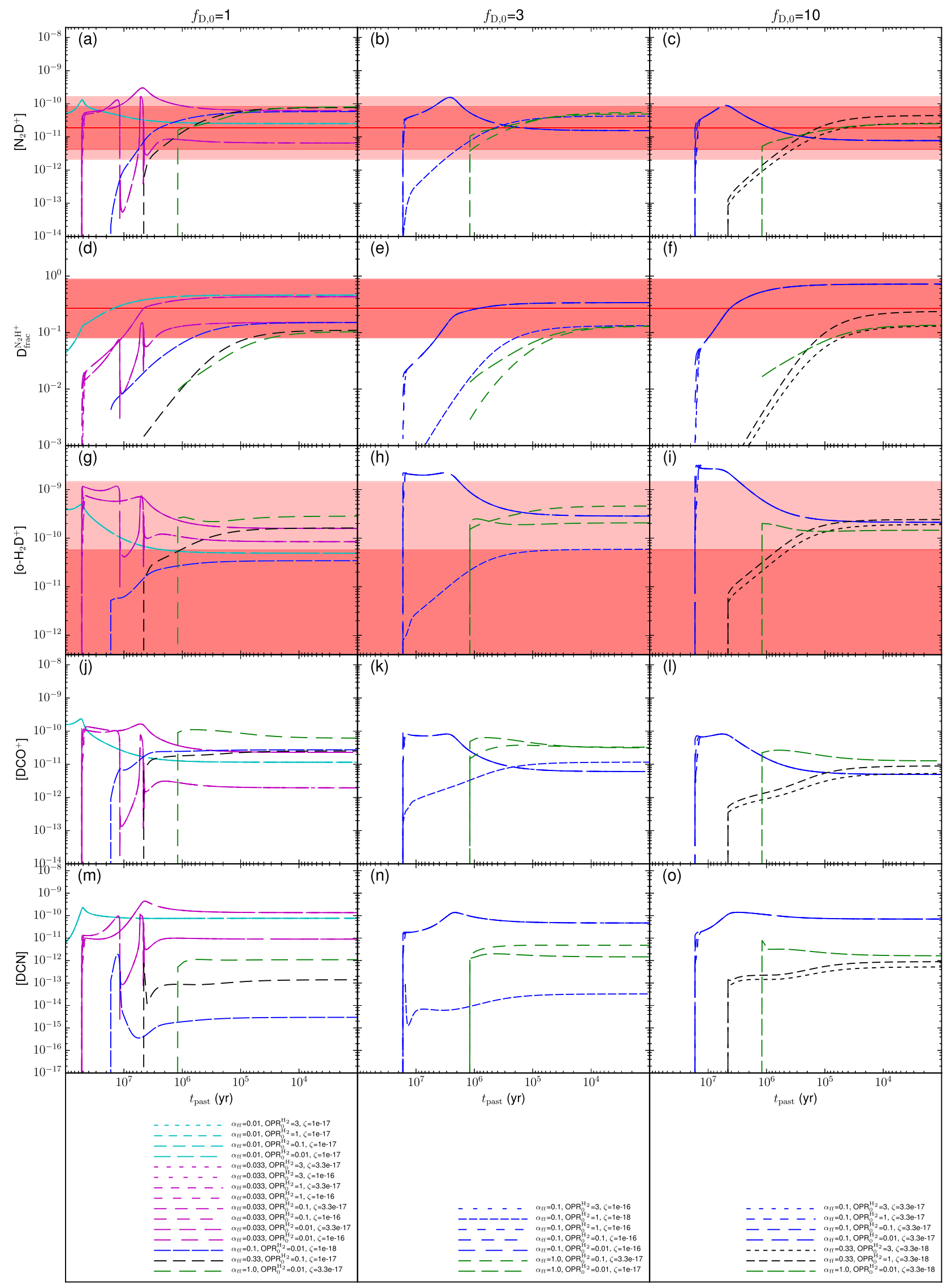

Figure 17. Most promising models for $\mathrm{C} 1-\mathrm{N}$ with $\delta n_{\mathrm{H}}^{\prime}=0.01$, following the format of Figure 16. 


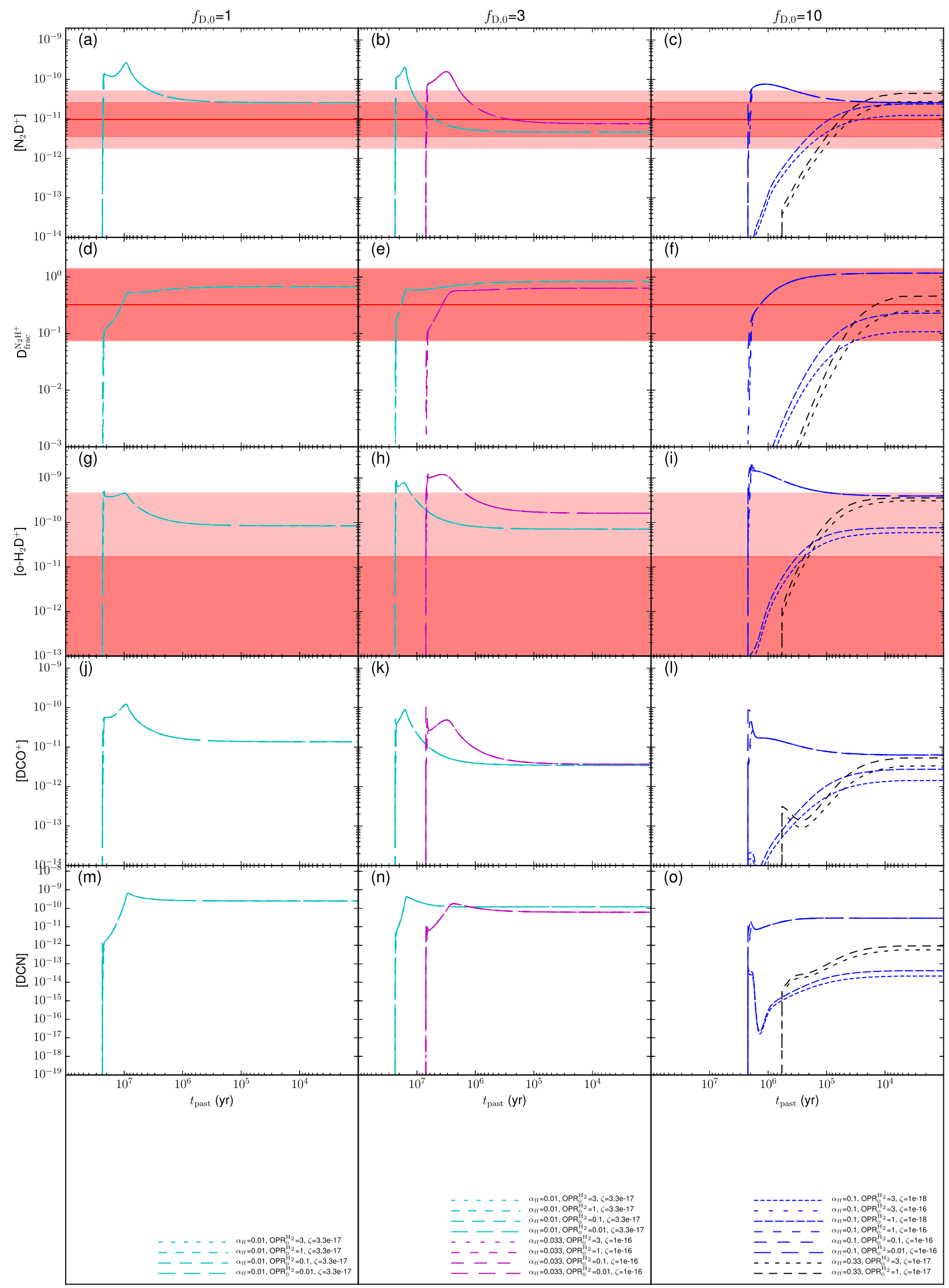

Figure 18. Most promising models for C1-S with $\delta n_{\mathrm{H}}^{\prime}=0.1$, following the format of Figure 16 . 


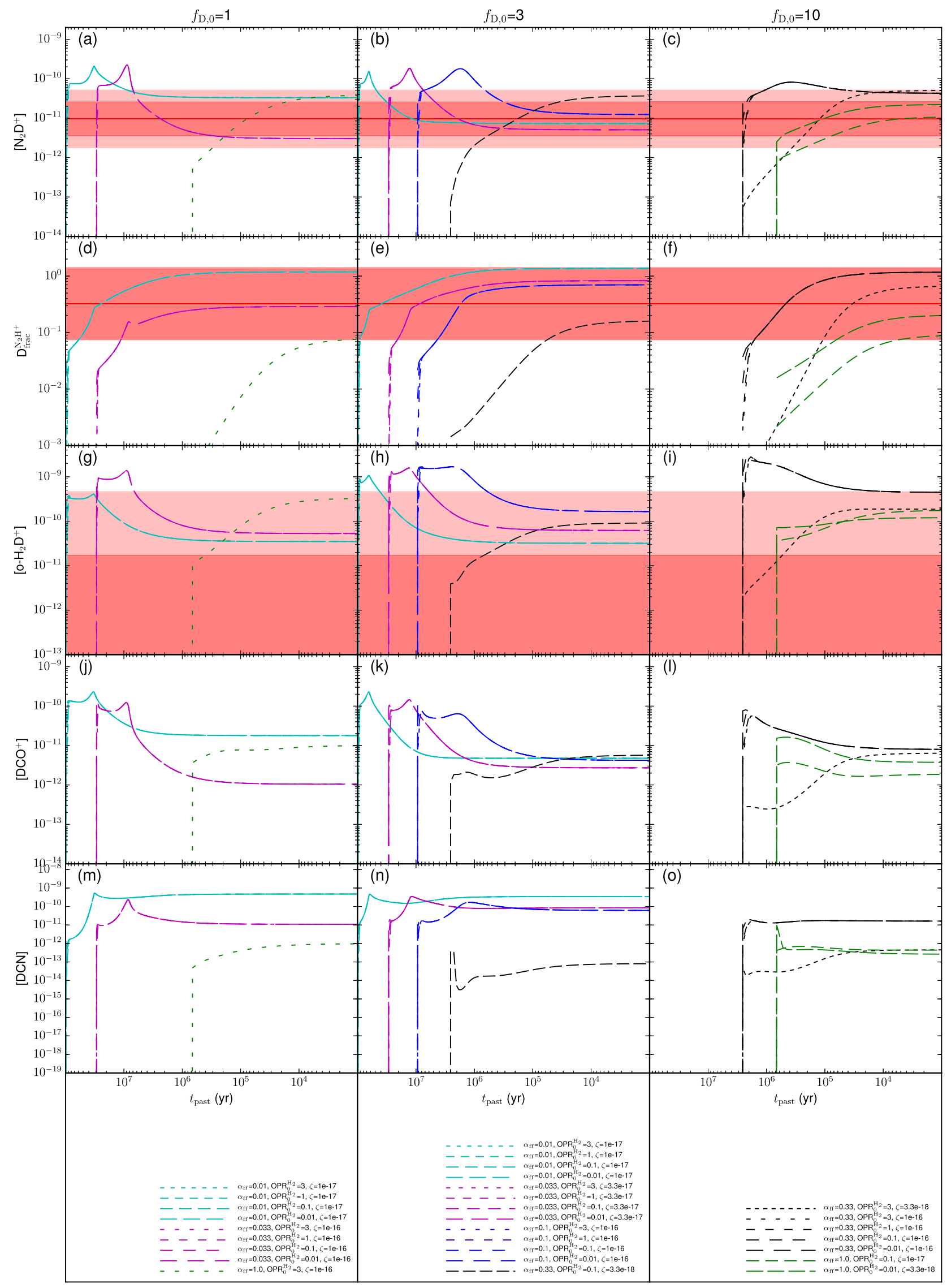

Figure 19. Most promising models for C1-S with $\delta n_{\mathrm{H}}^{\prime}=0.01$, following the format of Figure 16. 
Determination of $\left[\mathrm{N}_{2} \mathrm{H}^{+}\right]$is subject to some ambiguity. The locations of C1-N and C1-S are not precisely coincident with $\mathrm{N}_{2} \mathrm{H}^{+}$peaks that are seen in the CARMA and SMA maps. In addition, we see an extended, continuous $\mathrm{N}_{2} \mathrm{H}^{+}$structure around the $\mathrm{N}_{2} \mathrm{D}^{+}$cores. Under such circumstances, it is quite uncertain what fraction of $\mathrm{N}_{2} \mathrm{H}^{+}$line flux is emitted from the $\mathrm{N}_{2} \mathrm{D}^{+}$cores, and in practice this has been a main contributor to the uncertainty in the $D_{\mathrm{frac}}^{\mathrm{N}_{2} \mathrm{H}^{+}}$measurement.

Fontani et al. (2011) have measured $D_{\text {frac }}^{\mathrm{N}_{2} \mathrm{H}^{+}}$in high-mass starless regions, including $\mathrm{C} 1$, to be $\gtrsim 0.4$, but did not resolve the structures. Miettinen et al. (2012) have measured $D_{\text {frac }}^{\mathrm{N}_{2} \mathrm{H}^{+}}$as high as $\sim 1.0$ in low-mass pre-stellar cores. Friesen et al. (2013) measured $D_{\text {frac }}^{\mathrm{N}_{2} \mathrm{H}^{+}}$in a sample of low-mass cores, with mean $D_{\text {frac }}^{\mathrm{N}_{2} \mathrm{H}^{+}}=0.08$ and maximum $D_{\text {frac }}^{\mathrm{N}_{2} \mathrm{H}^{+}}=0.2$. These values are comparable to our $D_{\text {frac }}^{\mathrm{N}_{2} \mathrm{H}^{+}}$measurements of C1-N and C1-S. These suggest that high values of $D_{\mathrm{frac}}^{\mathrm{N}_{2} \mathrm{H}^{+}}(\gtrsim 0.1)$ can be presented in both low-mass and high-mass cores. However, considering the shorter free-fall time in high-mass cores (typically they have a factor of $\gtrsim 10$ higher density), the question of how they are supported long enough to build up high $D_{\mathrm{frac}}^{\mathrm{N}_{2} \mathrm{H}^{+}}$becomes more intriguing. As discussed earlier, magnetic fields may play an important role here in slowing down their collapse.

\subsection{The Importance of $\mathrm{o}-\mathrm{H}_{2} \mathrm{D}^{+}$}

As can be seen from the results, $\mathrm{o}-\mathrm{H}_{2} \mathrm{D}^{+}$can place strong constraints on the modeling. As one of the first products in deuterium fractionation, $\mathrm{o}-\mathrm{H}_{2} \mathrm{D}^{+}$is probably the best observable deuterated species that gives clues about the progress of deuteration. For instance, in the fiducial case (Figures 8 and 9), those models with high enough $D_{\text {frac }}^{\mathrm{N}_{2} \mathrm{H}^{+}}$predict values of [o$\left.\mathrm{H}_{2} \mathrm{D}^{+}\right]$that are close to the current observational upper limits. Future, more sensitive observations of $\mathrm{o}-\mathrm{H}_{2} \mathrm{D}^{+}$should play a key role in breaking the degeneracies among the currently allowed models.

\section{CONCLUSIONS}

We have measured the deuterium fraction $D_{\text {frac }}^{\mathrm{N}_{2} \mathrm{H}^{+}}$in two massive starless/early-stage cores (C1-N and C1-S) first identified by Tan et al. (2013). To do this, multiple transitions of $\mathrm{N}_{2} \mathrm{D}^{+}$and $\mathrm{N}_{2} \mathrm{H}^{+}$lines were observed with ALMA, CARMA, SMA, JCMT, NRO $45 \mathrm{~m}$, and IRAM $30 \mathrm{~m}$ telescopes. These data reveal interesting, disturbed kinematics around the cores and also indicate the presence of significant $\mathrm{N}_{2} \mathrm{H}^{+}$emission from the clump envelope, including a relatively warm component. Still, by considering a model of emission from the $\mathrm{N}_{2} \mathrm{D}^{+}$(3-2)-defined cores, excitation temperatures, $T_{\mathrm{ex}}$, and column densities and abundances of $\mathrm{N}_{2} \mathrm{D}^{+}$and $\mathrm{N}_{2} \mathrm{H}^{+}$in the cores were estimated by simultaneously fitting all available spectra.

Astrochemical models of collapsing cores have been run with a variety of initial conditions. The main parameter of our interest is the collapse rate, $\alpha_{\mathrm{ff}}$. However, results can also depend on the cosmic-ray ionization rate $\zeta$, initial density relative to final density $\mathrm{n}_{H, 0} / \mathrm{n}_{H, 1}$, initial depletion factor $f_{D, 0}$, and initial ortho-to-para $\mathrm{H}_{2}$ ratio $\mathrm{OPR}_{0}^{\mathrm{H}_{2}}$. Comparison between the observations and the models suggests that the most favorable models have $\alpha_{\mathrm{ff}}<0.33$ for both C1-N and C1-S, including many models with $\alpha_{\mathrm{ff}} \ll 1$, so that there is sufficient time for chemical equilibrium to be established. The few fast- collapse models that are consistent with the data require small initial values of $\mathrm{OPR}^{\mathrm{H}_{2}}$, which in itself indicates a chemically evolved cloud as the starting condition for core formation.

Our study has shown that the measurement of $D_{\text {frac }}^{\mathrm{N}^{2} \mathrm{H}^{+}}$and [o$\mathrm{H}_{2} \mathrm{D}^{+}$] can provide powerful constraints on the dynamics of massive starless/early-stage cores. However, improved observations, especially of $\left[0-\mathrm{H}_{2} \mathrm{D}^{+}\right]$and other deuterated species are needed to disentangle certain degeneracies among the allowed models.

We thank an anonymous referee for helpful comments. We thank Jan Wouterloot for helping with the JCMT observation. S.K. and J.C.T. acknowledge an NRAO/SOS grant and NSF grant AST 1411527. P.C. acknowledges the financial support of the European Research Council (ERC; project PALs 320620). This paper makes use of the following ALMA data: ADS/JAO.ALMA\#2011.0.00236.S. ALMA is a partnership of ESO (representing its member states), NSF (USA) and NINS (Japan), together with NRC (Canada), NSC and ASIAA (Taiwan), and KASI (Republic of Korea), in cooperation with the Republic of Chile. The Joint ALMA Observatory is operated by ESO, AUI/NRAO, and NAOJ. Support for CARMA construction was derived from the states of California, Illinois, and Maryland, the James S. McDonnell Foundation, the Gordon and Betty Moore Foundation, the Kenneth T. and Eileen L. Norris Foundation, the University of Chicago, the Associates of the California Institute of Technology, and the National Science Foundation. Ongoing CARMA development and operations are supported by the National Science Foundation under a cooperative agreement (NSF AST 08-38226) and by the CARMA partner universities. The James Clerk Maxwell Telescope has historically been operated by the Joint Astronomy Center on behalf of the Science and Technology Facilities Council of the United Kingdom, the National Research Council of Canada and the Netherlands Organization for Scientific Research. We are grateful to the staff members at the Nobeyama Radio Observatory (NRO) for both operating the $45 \mathrm{~m}$ and helping us with the data reduction; NRO is a branch of the National Astronomical Observatory, National Institutes of Natural Sciences, Japan. The National Radio Astronomy Observatory is a facility of the National Science Foundation operated under cooperative agreement by Associated Universities, Inc.

Facilities: ALMA, CARMA, SMA, JCMT, No:45m, IRAM:30m.

\section{REFERENCES}

Bergin, E. A., \& Tafalla, M. 2007, ARA\&A, 45, 339

Bonnell, I. A., Bate, M. R., Clarke, C. J., \& Pringle, J. E. 2001, MNRAS, 323, 785

Brünken, S., Sipilä, O., Chambers, E. T., et al. 2014, Natur, 516, 219

Butler, M. J., \& Tan, J. C. 2009, ApJ, 696, 484

Butler, M. J., \& Tan, J. C. 2012, ApJ, 754, 5

Caselli, P., Myers, P. C., \& Thaddeus, P. 1995, ApJL, 455, L77

Caselli, P., van der Tak, F. F. S., Ceccarelli, C., \& Bacmann, A. 2003, A\&A, 403, L37

Caselli, P., Walmsley, C. M., Tafalla, M., Dore, L., \& Myers, P. C. 1999, ApJL, 523, L165

Caselli, P., Walmsley, C. M., Zucconi, A., et al. 2002, ApJ, 565, 344

Chambers, E. T., Jackson, J. M., Rathborne, J. M., \& Simon, R. 2009, ApJS, 181,360

Chen, H.-R., Liu, S.-Y., Su, Y.-N., \& Zhang, Q. 2010, ApJL, 713, L50

Crabtree, K. N., Indriolo, N., Kreckel, H., Tom, B. A., \& McCall, B. J. 2011, ApJ, 729, 15

Crapsi, A., Caselli, P., Walmsley, C. M., et al. 2005, ApJ, 619, 379 
Crapsi, A., Caselli, P., Walmsley, M. C., \& Tafalla, M. 2007, A\&A, 470, 221 Emprechtinger, M., Caselli, P., Volgenau, N. H., Stutzki, J., \& Wiedner, M. C. 2009, A\&A, 493, 89

Fontani, F., Busquet, G., Palau, A., et al. 2015, A\&A, 575, A87

Fontani, F., Palau, A., Caselli, P., et al. 2011, A\&A, 529, L7

Friesen, R. K., Di Francesco, J., Bourke, T. L., et al. 2014, ApJ, 797, 27

Friesen, R. K., Kirk, H. M., \& Shirley, Y. L. 2013, ApJ, 765, 59

Girart, J. M., Beltrán, M. T., Zhang, Q., Rao, R., \& Estalella, R. 2009, Sci, 324,1408

Hernandez, A. K., Tan, J. C., Caselli, P., et al. 2011, ApJ, 738, 11

Hernandez, A. K., Tan, J. C., Kainulainen, J., et al. 2012, ApJL, 756, L13

Kong, S., Caselli, P., Tan, J. C., Wakelam, V., \& Sipilä, O. 2015, ApJ, 804, 98

McKee, C. F. \& Ostriker, E. C. 2007, ARA\&A, 45, 565

McKee, C. F., \& Tan, J. C. 2002, Natur, 416, 59

McKee, C. F., \& Tan, J. C. 2003, ApJ, 585, 850

Miettinen, O., Harju, J., Haikala, L. K., \& Juvela, M. 2012, A\&A, 538, A137

Padovani, M., \& Galli, D. 2011, A\&A, 530, A109

Pagani, L., Lesaffre, P., Jorfi, M., et al. 2013, A\&A, 551, A38

Pagani, L., Salez, M., \& Wannier, P. G. 1992, A\&A, 258, 479

Pillai, T., Kauffmann, J., Tan, J. C., et al. 2015, ApJ, 799, 74
Pillai, T., Wyrowski, F., Carey, S. J., \& Menten, K. M. 2006, A\&A, 450, 569

Pon, A., Caselli, P., Johnstone, D., et al. 2015, A\&A, 577, A75

Rathborne, J. M., Jackson, J. M., \& Simon, R. 2006, ApJ, 641, 389

Shu, F. H., Adams, F. C., \& Lizano, S. 1987, ARA\&A, 25, 23

Simon, R., Jackson, J. M., Rathborne, J. M., \& Chambers, E. T. 2006, ApJ, 639, 227

Sipilä, O., Caselli, P., \& Harju, J. 2013, A\&A, 554, AA92

Sipilä, O., Caselli, P., \& Harju, J. 2015, A\&A, 578, A55

Tan, J. C., Beltrán, M. T., Caselli, P., et al. 2014, in Protostars and Planets VI, ed. H. Beuther et al. (Tucson, AZ: Univ. of Arizona Press), 149

Tan, J. C., Kong, S., Butler, M. J., Caselli, P., \& Fontani, F. 2013, ApJ, 779, 96

van der Tak, F. F. S., Black, J. H., Schöier, F. L., Jansen, D. J., \& van Dishoeck, E. F. 2007, A\&A, 468, 627

Wang, P., Li, Z.-Y., Abel, T., \& Nakamura, F. 2010, ApJ, 709, 27

Wang, Y., Zhang, Q., Rathborne, J. M., Jackson, J., \& Wu, Y. 2006, ApJL, 651, L125

Ward-Thompson, D., André, P., Crutcher, R., et al. 2007, in Protostars and Planets V, ed. B. Reipurth, D. Jewitt, \& K. Keil (Tucson, AZ: Univ. of Arizona Press), 33

Zhang, Q., Qiu, K., Girart, J. M., et al. 2014, ApJ, 792, 116 